\title{
Mechanical Properties of Miura-Based Folded Cores under Quasi-Static Loads
}

\author{
Xiang Zhou ${ }^{1 *}$, Hai Wang ${ }^{1}$, Zhong You ${ }^{2}$ \\ 1 School of Aeronautics and Astronautics, Shanghai Jiao Tong University, China \\ 2 Department of Engineering Science, University of Oxford, UK
}

\begin{abstract}
Sandwich structures with folded cores are regarded as a promising alternative to conventional honeycomb sandwich structures in the aerospace industry. This paper presents a parametric study on the mechanical properties of a variety of Miura-based folded core models virtually tested in quasi-static compression, shear and bending using the finite element method. It is found that the folded core models with curved fold lines exhibit the best mechanical performances in compression and shear while the multiple-layered models outperform the other folded core models in bending. Furthermore, the folded core models are compared to a honeycomb core model with the same density and height. In this case, it is shown that the honeycomb core has the best performance in compression while the folded cores have comparable or even better performances in the shear and bending cases. The virtual test results reported in this paper can provide researchers with a general guideline to design the most suitable folded core structure for a certain application.
\end{abstract}

Keywords: Miura-based folded core; sandwich structure; quasi-static test; finite element method.

\footnotetext{
${ }^{*}$ Corresponding author, E-mail: xiangzhou@sjtu.edu.cn, phone: +86-21-34207538, address: School of Aeronautics and Astronautics, No. 800 Dongchuan Road, Shanghai, 200240, China. X. Zhou is an associate professor in the School of Aeronautics and Astronautics at Shanghai Jiao Tong University.
} 


\section{INTRODUCTION}

Composite sandwich structures, typically consisting of two thin and stiff faces separated by a thick lightweight cellular core, have many successful applications in the aerospace industry where weight-saving is a paramount design goal. In this context, honeycomb cores made of aluminum or Nomex paper are the most commonly used core type today due to their excellent weight-specific mechanical properties. However, honeycomb cores are known to suffer from an undesirable moisture accumulation problem whereby the condensed moisture is trapped inside the sealed hexagon cells leading to deterioration of the mechanical performance over time [1].

Folded cores, made by folding sheet material into a three-dimensional structure according to the principle of origami - an ancient art of paper folding, do not have the moisture accumulation problem because of the existence of open channels in such structures. Moreover, they allow for tailored mechanical properties with a wide range of possible configurations. Therefore, they emerge as a promising alternative to conventional honeycomb cores and have seen a surge in research interest from the aerospace industry in recent years. For example, in the transnational project CELPACT, the fabrication cost and impact performance of three different advanced cellular core concepts, i.e. folded core, selected laser melted lattice core were evaluated and compared [2]. Besides, the aircraft manufacturer Airbus presented a sandwich fuselage concept VeSCo which incorporates folded cores as a sandwich core material [3] and has made a $4.5 \mathrm{~m}^{2}$ test assembly consisting of approximate 165,000 creases [4].

While specimen manufacturing and mechanical testing remain routine procedures, numerical analysis based on the finite element (FE) method, as an established time- and cost-efficient tool, has been widely adopted in the development of new composite structures. Besides, FE simulations can provide analysis details such as the cross-sectional stress/strain data that are usually difficult to be obtained experimentally. As a result, a number of numerical studies of folded-core sandwich structures, such as virtual in- and out-of-plane quasi-static compression and shear tests [5-9], lowand high-velocity impact simulations [10-12], residual bending strength simulations after impact [13] and macro- and multi-scale modelling [7,11], are available in the literature. However, most folded cores used in research works are made of two simple Miura-based unit cell geometries with 
zigzag and chevron shapes [14]. So far, the authors are not aware of any literature on computational or experimental study of folded core structures beyond these two simple cases. Consequently, the mechanical properties of other folded configurations remain unexplored.

This paper presents a parametric study on folded cores with different geometric parameters based on the standard Miura folding pattern [15] and its variation forms subject to out-of-plane compression, in-plane shear and bending using the finite element method. To facilitate the parametric modelling, a new origami geometric design approach, known as the vertex method [16], is used to generate the various folded core models in this study. Furthermore, the weight-specific mechanical properties of the folded core models were compared to those of a honeycomb model with the same density.

The layout of the paper is arranged as follows. First, the mechanical behaviors of eight folded core models with the standard Miura origami folding pattern are simulated and compared. Second, eight folded core models with curved fold lines are virtually tested. Third, further two folded core models with multiple layers are considered. Fourth, the mechanical performances of the folded core models are compared with those of a honeycomb core model. Finally, a brief discussion concludes the paper.

\section{STANDARD MIURA FOLDED CORES}

\subsection{Geometric models}

Using a set of geometric parameters to define the folded configuration of a unit cell is a commonly employed modelling technique of folded cores in the literature [4]. However, this approach lacks flexibility in that a new set of geometric parameters and their relationships must be established when a different type of folded core is studied. In this paper, an alternative modelling technique, known as the vertex method for designing developable origami structures, is used to generate the geometric models of folded cores, in which $m$ input points in the $x$-z plane, denoted by their position vectors $V_{i}^{x}=\left[\begin{array}{ccc}x_{i}^{x} & 0 & z_{i}^{x}\end{array}\right]^{\mathrm{T}}, i=1,2, \ldots, m$, and $n+2$ input points in the $y$-z plane, 
denoted by their position vectors $\boldsymbol{V}_{j}^{y}=\left[\begin{array}{lll}0 & y_{j}^{y} & z_{j}^{y}\end{array}\right]^{\mathrm{T}}, j=0,1, \ldots, n+1$ are first specified in a Cartesian coordinate system, respectively and then $m \times n$ vertices $\boldsymbol{V}_{\boldsymbol{i}, \boldsymbol{j}}$ of the target folded core geometric model are obtained using the following equation

$$
\boldsymbol{V}_{\boldsymbol{i}, \boldsymbol{j}}=\left[\begin{array}{l}
x_{i, j} \\
y_{i, j} \\
z_{i, j}
\end{array}\right]=\boldsymbol{V}_{\boldsymbol{j}}^{\boldsymbol{y}}+\left[\boldsymbol{A}_{\boldsymbol{j}}\right] \boldsymbol{V}_{\boldsymbol{i}}^{\boldsymbol{x}}, i=1,2, \ldots, m ; j=1,2, \ldots, n,
$$

where $\left[\boldsymbol{A}_{\boldsymbol{j}}\right]$ is a $3 \times 3$ matrix given by

$$
\left[\mathrm{A}_{j}\right]=\left[\begin{array}{ccc}
1 & 0 & 0 \\
0 & 0 & (-1)^{j} \frac{\cos \theta_{j-1}+\cos \theta_{j}}{\sin \left(\theta_{j-1}-\theta_{j}\right)} \\
0 & 0 & (-1)^{j} \frac{\sin \theta_{j-1}+\sin \theta_{j}}{\sin \left(\theta_{j-1}-\theta_{j}\right)}
\end{array}\right],
$$

where the angular variable $\theta_{j}$ is determined by

$$
\begin{aligned}
& \sin \theta_{j}=\frac{i_{z} \cdot\left(V_{j+1}^{y}-V_{j}^{y}\right)}{\left\|V_{j+1}^{y}-V_{j}^{y}\right\|}, \\
& \cos \theta_{j}=\frac{i_{y} \cdot\left(V_{j+1}^{y}-V_{j}^{y}\right)}{\left\|V_{j+1}^{y}-V_{j}^{y}\right\|^{\prime}},
\end{aligned}
$$

where $\boldsymbol{i}_{\boldsymbol{y}}=\left[\begin{array}{lll}0 & 1 & 0\end{array}\right]^{\mathrm{T}}$ and $\boldsymbol{i}_{\boldsymbol{z}}=\left[\begin{array}{lll}0 & 0 & 1\end{array}\right]^{\mathrm{T}}$ are the unit vectors of the $y$ and $z$ axis, respectively, and $\|\boldsymbol{u}\|$ denotes the norm of a vector $\boldsymbol{u}$.

Figure 1 shows the input points in the $x-z$ and $y-z$ planes used to generate the models in this section, which are defined by four parameters, i.e. $\alpha, h_{x}, \beta$ and $h_{y}$. By fixing $h_{y}$ to $10 \mathrm{~mm}$ and choosing different combinations of values for the other three parameters, eight unit cell models known as standard Miura origami structures are obtained, as shown in Fig. 2. The core density $\rho_{c}$ can be obtained by

$$
\rho_{c}=\frac{t_{m} S_{m}}{V_{u}} \rho_{m}
$$

where $t_{m}, S_{m}$ and $\rho_{m}$ are respectively the thickness, total area and material density of the sheet from which a unit cell of the core is folded and $V_{u}$ is the spatial volume of the unit cell, defined by

$$
V_{u}=S_{u} H_{c}
$$

where $S_{u}$ is the base area of the unit cell and $H_{c}$ is the core height, as illustrated in Fig. 3. Since the weight-specific mechanical properties of the folded cores are concerned, a unified core density equal to $0.05 \rho_{m}$ is used for all models studied in this paper. According to Eqn. (5), the thickness 
of the sheet material is given by

$$
t_{m}=0.05 \frac{V_{u}}{S_{m}} .
$$

Table 1 summarizes the geometric properties of the eight unit cell models considered in this section. It is found that $t_{m}$ is not affected by $h_{x}$ given the other input parameters are fixed. The larger $h_{x}$ is, the larger the amplitude of the flatwise zigzag fold lines is. With the increase in $\alpha$ or $\beta, t_{m}$ becomes smaller whereas the folded core becomes denser in the $x$ - or $y$-direction.

\subsection{FE models}

The finite element analysis was performed in the FE solver ABAQUS/Explicit (SIMULIA Inc., USA) due to its good capability to cope with large nonlinear deformations, post-buckling behaviors and complex contact conditions. Because the main purpose of this paper is to study the structural influence on the mechanical properties of folded cores, both the faces and the core are assumed to be made of 5052-O aluminum alloy and a bilinear isotropic plastic material model [17] is employed for simplicity. For quasi-static loading cases, the strain rate effect is not considered. The detailed material parameters are summarized in Table 2 .

$\mathrm{S} 4 \mathrm{R}$, the four-node quadrilateral shell element with reduced integration and hourglass control, is the element of choice in the simulation. With this particular element type, the mesh density has a strong influence on the accuracy of the simulation results. Although a coarser mesh reduces the computational time, it is not able to accurately represent the post-buckling behavior of the facets. Therefore, convergence testing of different element sizes ranging from $0.15 \mathrm{~mm}$ to $0.4 \mathrm{~mm}$ was firstly performed for all eight unit cell models in Fig. 2 subject to compressive loads in the thickness direction. The results converged for element sizes below $0.2 \mathrm{~mm}$. Therefore, the $0.2 \mathrm{~mm}$ element size is used for all subsequent analysis unless otherwise specified. In the virtual tests, each folded core model consists of four unit cells in a $2 \times 2$ array, as shown in Fig. 4. The numbers of elements in the eight folded core models used in the virtual tests range from 29696 for M17 to 98832 for M12.

Three types of virtual tests, i.e. compression, shear and bending, were considered. In the virtual 
compression test, two rigid plates RP1 and RP2, parallel to the $x-y$ plane, were attached to two ends of the model in the thickness direction using the tie constraint, as shown in Fig. 5(a). The general contact algorithm was employed to model the self-contact of the folded core and the surface-to-surface contact between the core and each rigid plate. Rigid plate RP1 was fixed both translationally and rotationally, and rigid plate RP2 was displaced half of the thickness, i.e. $5 \mathrm{~mm}$, towards RP1, resulting in a maximum loaded compressive strain of $50 \%$. The loading rate was chosen as $500 \mathrm{~mm} / \mathrm{s}$ to ensure quasi-static results while allowing the simulation to complete within a reasonable computational time. In the virtual shear test, the same model as that in the compression test was used, as shown in Figs. 5(b-c). Rigid plate RP1 was still completely fixed. In two load cases, rigid plate RP2 was displaced 5-mm in the $y$ - and $x$-directions, respectively with its translational degree of freedom in the thickness direction unconstrained, resulting in a maximum loaded shear strain of $50 \%$. The loading rate was still set as $500 \mathrm{~mm} / \mathrm{s}$ to ensure the simulation was conducted quasi-statically. In the virtual bending test, the two rigid plates used in the previous tests were replaced by two 1-mm thick faces made of the same material as the core to form a sandwich structure, as shown in Fig. 6. The faces are meshed with S4R elements with the same element size for the core. Two load cases were performed separately. In the first load case, two rigid plates RP3 and RP4 were attached to two $y$-directional ends of the model, as shown in Fig. 6(a). The two rigid plates were rotated about the $x$ axis by $0.01 l_{y}$ and $-0.01 l_{y}$, respectively to bend the sandwich structure in the $x$-direction to a resultant curvature of 0.02 . In the second load case, two rigid plates RP5 and RP6 were attached to the $x$-directional ends of the model, as shown in Fig. 6(b). Then, they were rotated about the $y$ axis by $0.01 l_{x}$ and $-0.01 l_{x}$, respectively to bend the sandwich structure in the $y$-direction to a resultant curvature of 0.02 . In both bending load cases, the loading rate was chosen as $100 \mathrm{rad} / \mathrm{s}$ to ensure quasi-static results.

\subsection{Results}

The effective compressive stress-strain curves of models M11 to M18 in the virtual compression test are plotted in Fig. 7 where a logarithmic scale is used for the strain axis to better illustrate regions of small strains. According to the curves, the behaviors of the folded cores under compression are characterized by three distinct stages, i.e. pre-buckling (stage I), folding (stage II) and densification (stage III). In the first stage, the folded cores behave linear-elastically up to an 
average strain of $0.06 \%$ where yield of the material begins to propagate. The compressive stiffnesses $E_{c}$ of the eight models in the linear-elastic range are listed in the second column of Table 3. It is noted that $E_{c}$ increases with the increase in $\alpha, h_{x}$ or $\beta$ and is more sensitive to the change of $\beta$ than to that of $\alpha$. In the second stage, buckling followed by folding of the facets occurs, which is accompanied by softening of the folded cores in the thickness direction. Since the facets do not buckle and fold at the same time, two sub-stages can be identified. Specifically, the facets in the outer two $x$-directional rows buckle and fold at first, as shown in Fig. 8(a), followed by buckling and folding of the facets in the middle two $x$-directional rows, as shown in Fig. 8(b). The third stage features densification of the folded cores where the facets come into contact with each other and/or with the rigid plates, as shown in Fig. 8(c), resulting in an increase in the compressive stress. Figure 9 compares the absorbed compressive energies per area, i.e. the surface under the stress-strain curve, of the eight models. It is noted that models with smaller $\alpha$ and $h_{x}$ absorb more compressive energy than those with larger $\alpha$ and $h_{x}$, and the energy absorption capacity is less sensitive to $\beta$.

Figures 10(a) and (b) show the effective shear stress-strain curves of models M11 to M18 in the virtual shear tests in the $y-z$ and $x-z$ planes, respectively. Similar to the compression loading case, three stages, i.e. pre-buckling, folding and densification, are observed in both of the shear loading cases while the folding stage can be further divided into sub-stage 1 - buckling and folding of the facets in the outer two $y$-directional rows and sub-stage 2 - buckling and folding of the facets in the middle two $y$-directional rows. The representative deformed shapes of model M11 in folding sub-stage 1 (at $2 \%$ strain), folding sub-stage 2 (at 20\% strain) and densification stage (at $50 \%$ strain) during shear loading in the $y$-z plane are shown as an example in Figs. 11(a), (b) and (c), respectively. The shear stiffnesses in the $y$-z plane $E_{s 1}$ and in the $x$-z plane $E_{s 2}$ of models M11 to M18 in the linear-elastic range are listed in the third and fourth columns of Table 3, respectively. In the $y$-z plane, the shear stiffness $E_{s 1}$ increases with the increase in $\alpha$ or $h_{x}$ but decreases with the increase in $\beta$. In the $x$-z plane, the shear stiffness $E_{s 2}$ increases with the increase in $h_{x}$ or $\beta$ but decreases with the increase in $\alpha$. Figures 12(a) and (b) compare the absorbed shear energies per area in the two shear loading cases. It is consistently observed in both cases that models with smaller $\alpha, h_{x}$ or $\beta$ absorb more shear energy than models with larger $\alpha, h_{x}$ or $\beta$ 
do. For the $y$-z plane loading case, $\beta$ has the greatest influence on the energy absorption capacity whereas for the $x$-z plane loading case, $\alpha$ plays a decisive role to the energy absorption capacity.

Figures 13(a) and (b) respectively show the bending moments in the $x$-direction normalized by $l_{x}$ and in the $y$-direction normalized by $l_{y}$ of models M11 to M18 plotted against the curvature. In both the $x$-directional and the $y$-directional bending cases, the bending moment first increases linearly with the curvature until yield of the material occurs, then continues to increase with a reduced slope up to buckling of the sandwich structure and finally decreases sharply afterwards. The pre-buckling (a) and post-buckling (b-c) deformed shapes of model M11 in the $x$ - and $y$-directional bending cases are shown in Figs. 14(a) and (b), respectively. It is noted that both cases involve buckling of both the folded core and the upper face. The bending stiffnesses in the linear-elastic range and the bending moments and curvatures at buckling point of models M11 to M18 are listed in Table 4. For the $x$-directional bending case, the bending stiffness $E I_{x}$ increases with the increase in $h_{x}$ or $\beta$. Both of the critical bending moment $M_{x}^{c r}$ and curvature $k_{x}^{c r}$ at buckling point increase with the increase in $\alpha$ or $\beta$. For the $y$-directional bending case, the bending stiffness $E I_{y}$ decreases with the increase in $\alpha, h_{x}$ or $\beta$. While the critical bending moment $M_{y}^{c r}$ and curvature $k_{y}^{c r}$ at buckling point also decreases with the increase in $h_{x}$, they increase with the increase in $\alpha$ or $\beta$ on the contrary.

\section{FOLDED CORES WITH CURVED FOLD LINES}

\subsection{Model description}

In this section, the influence of curved fold lines on the mechanical properties of folded cores is investigated. According to discussion in section 2.3, model M11 has overall the best energy absorption performances under compressive and shear loads. Therefore, folded core structures with curved fold lines that are modified from model M11 are considered.

The $x$-z plane inputs used to generate the unit cell models in this section are shown in Fig. 15 where the input points $V_{i}^{x}, i=1,2, \ldots$ are densely located on the solid curved line consisting of four sub-segments each of which is a part of a circle and tangential to the solid-dotted lines at 
intersection points. The $y$-z plane inputs are the same as those of model UM11 and hence are not shown here. It is noted that the unit cell geometry can be totally determined by a single parameter $\gamma$. When $\gamma$ is equal to zero, model UM11 is gained. By varying $\gamma$ from $\pi / 2$ to $-\pi / 2$, eight unit cell models are generated. The larger the absolute value of $\gamma$ is, the more curved the fold line is. The geometric properties of these models are listed in Table 5. It is noted that the wall thickness $t_{m}$ increases with the decrease in the absolute value of $\gamma$ and models with the same absolute value of $\gamma$ have the same $t_{m}$.

The eight folded core models M21 to M28 used in the virtual tests in this section are shown in Fig. 16 , each of which consists of four unit cells in a $2 \times 2$ array. An average element size of $0.2 \mathrm{~mm}$ is used to mesh the models according to the mesh convergence tests. The numbers of elements in the eight models range from 49984 to 54528 . The same virtual tests as described in the previous section, i.e. compression in the $z$-direction up to $50 \%$ compressive strain, shear in the $y-z$ and $x-z$ planes up to $50 \%$ shear strain and bending in the $x$ - and $y$-direction up to a resultant curvature of $0.02 \mathrm{~mm}^{-1}$ were performed.

\subsection{Results}

For the virtual compression test, Fig. 17 illustrates the effective compressive stress versus strain curves of models M21 to M28 and M11 and the corresponding absorbed energy versus strain curves are shown in Fig. 18. The compressive stiffness $E_{c}$ and ultimate strength $\sigma_{c}^{c r}$ of the nine models are listed in the second and third columns of Table 6, respectively. It is noted that $E_{c}$ increases with the increase in $\gamma$ while $\sigma_{c}^{c r}$ increases with the absolute value of $\gamma$. Models with positive $\gamma$ show better energy absorption performances in compression than models with negative $\gamma$. All models with curved fold lines exhibit improved compressive performances than M11.

The effective shear stress-strain curves and the corresponding absorbed shear energy-strain curves of models M21 to M28 and M11 in the y-z plane are shown in Figs. 19(a) and 20(a), respectively. The shear stiffness $E_{s 1}$ and ultimate strength $\sigma_{s 1}^{c r}$ in the $y$-z plane of the nine models are listed in the fourth and fifth columns of Table 6, respectively. It is noted that $E_{s 1}$ of the nine models are 
basically the same, all within $0.8 \%$ deviation to that of M11. $\sigma_{s 1}^{c r}$ increases with the increase in the absolute value of $\gamma$. Models with positive $\gamma$ in general have better shear energy absorption performances in the $y-z$ plane than models with negative $\gamma$. Again, all models with curved fold lines absorb more shear energy in the $y$-z plane than M11 does.

The $x$-z plane counterparts of Figs. 19(a) and 20(a) are drawn in Figs. 19(b) and 20(b), respectively, and the shear stiffness $E_{s 2}$ and ultimate strength $\sigma_{s 2}^{c r}$ in the $x$-z plane of the nine models are listed in the last two columns of Table 6. In this case, however, the shear stiffness $E_{s 2}$ decreases with the increase in the absolute value of $\gamma$. While M11 has the highest stress-strain curve before the ultimate shear strength $\sigma_{s 2}^{c r}$ is reached, models M21-M23 and M26-M28 outperforms M11 afterwards. In general, models with negative $\gamma$ exhibit better shear performance in the $x$-z plane than models with positive $\gamma$.

For the virtual bending tests, the bending moment per $l_{x}$ versus curvature curves in the $x$ - and $y$-directions of models M21 to M28 and M11 are plotted in Figs 21(a) and (b), respectively. Their bending stiffness $E I_{x}$ or $E I_{y}$ and maximum bending moment $M_{x}^{c r}$ or $M_{y}^{c r}$ in the $x$ - and $y$-directions are listed in Table 7. It is noted that for the $x$-directional bending case, both $E I_{x}$ and $M_{x}^{c r}$ increase as $\gamma$ increases while in the $y$-directional case, the maximum bending moment $M_{y}^{c r}$ increases with the increase in $\gamma$ and the bending stiffness $E I_{y}$ of the nine models are literally the same.

\section{MULTIPLE-LAYERED FOLDED CORES}

\subsection{Model description}

In this section, two folded core models with multiple layers of materials M31 and M32 are considered. Model M31 consists of two identical layers where the fold lines on the bottom side of the upper layer and those on the top side of the lower layer are aligned and merged, as show in Fig. 22(a). Each layer contains eight unit cells in a $2 \times 4$ array, each of which is scaled from the unit cell model of M21 by a scaling factor of $50 \%$. The wall thicknesses of the upper and bottom layers are the same. Model M32 is a three-layered configuration with an additional flat sheet as the middle 
layer added between the upper and lower layers of model M31, as shown in Fig. 22(b), where the wall thicknesses of the upper, middle and lower layers are the same. The geometric properties of models M31 and M32 are summarized in Table 8. Both models are meshed with S4R elements with an average size of $0.2 \mathrm{~mm}$, resulting in 53760 elements for model M31 and 64939 for model M32.

\subsection{Results}

The compressive stress-strain curves of models M11, M21, M31 and M32 are shown by black dotted, black dash-dotted, blue solid and blue dashed lines in Fig. 23, respectively. Incidentally, the same line types are assigned to models M11, M21, M31 and M32 in all subsequent figures. The shear stress-strain curves in the $y-z$ and $x-z$ planes are shown in Figs. 24(a) and (b), respectively. The bending moment per $l_{x}$ or $l_{y}$ versus curvature curves in $x$-and $y$-directions are plotted in Figs. 25(a) and (b), respectively.

It is noted that the multiple-layered models M31 and M32 have the lower stress-strain curves than model M21 in compression and shear but they still outperforms model M11 in compression and shear in the $y-z$ plane soon after initial buckling. In terms of bending, the maximum bending moments of models M31 and M32 are much higher than those of model M21 in both the $x$ - and $y$-directional bending cases. Model M31 consistently outperforms model M32 in all five loading cases.

\section{COMPARISON WITH HONEYCOMB CORE}

In this section, the mechanical performances of the folded cores are compared to a honeycomb core with the same density i.e. $0.05 \rho_{m}$ and the same height i.e. $10 \mathrm{~mm}$. Figure 26(a) shows the unit cell geometries of the honeycomb model whose hexagon cell size is $2.5 \mathrm{~mm}$, leading to a wall thickness of $0.0812 \mathrm{~mm}$. The virtually tested honeycomb core model MHC consists of four unit cells in a $2 \times 2$ array, as shown in Fig. 26(b) and is meshed with the S4R elements with an average size of $0.08 \mathrm{~mm}$ according to the mesh convergence study. The total number of elements in the model equals 93600 . 
The compressive stress-strain curve of the honeycomb model MHC is shown by the red solid line in Fig. 23. The shear stress-strain curves in the $y-z$ and $x-z$ planes are drawn as the red solid lines in Figs. 24(a) and (b), respectively. The bending moment per $l_{x}$ or $l_{y}$ versus curvature curves in the $x$ - and $y$-directions are illustrated by the red solid lines in Figs. 25(a) and (b), respectively.

When compared to folded core models M21 and M31, the honeycomb model outperforms the folded core models in the compression and bending in the $x$-direction cases while the folded core models have comparable or even better performances than the honeycomb core for the rest cases.

\section{DISCUSSIONS AND CONCLUSIONS}

Three types of folded cores namely 1) standard Miura, 2) with curved fold lines and 3) multiple layered with the same density and height are virtually tested under five loading cases, i.e. out-of-plane compression, shear in the $y$-z and $x$-z planes, bending in the $x$ - and $y$-directions. The Vertex Method for designing three-dimensional origami structures is adopted to parameterize the geometric models of the folded cores.

For the standard Miura folded cores, the following conclusions can be reached: a) the sparser models (corresponding to smaller $\alpha$ and $\beta$ ) with smaller amplitude of the flatwise zigzag fold lines (corresponding to smaller $h_{x}$ ) exhibit better post-buckling and energy absorption performances in compression and shear where $\alpha$ plays the decisive role in the energy absorption capacity in the $x$-z plane shear case and the energy absorption capacity in the $y$-z plane shear case is mainly determined by $\beta$; b) the denser models with larger amplitude of the flatwise zigzag fold lines have higher compressive stiffness; c) the shear stiffness in the $y$-z plane increases with the increases in $\alpha$ and $h_{x}$ but the decrease in $\beta$ whereas the shear stiffness in the $x-z$ plane increases with the increase in $\beta$ and $h_{x}$ but the decrease in $\alpha$; d) the denser the model is in the $y$-direction (corresponding to larger $\beta$ ), the higher the $y$-directional bending stiffness and maximum bending moment are; e) the denser the model is in the $x$-direction (corresponding to larger $\alpha$ ), the higher the $x$-directional bending stiffness and maximum bending moment are.

Folded cores with both positively (i.e. positive $\gamma$ ) and negatively (i.e. negative $\gamma$ ) curved fold 
lines show better mechanical performances than the corresponding standard Miura folded core in compression and shear in the $y$-z plane whereas the folded cores with positively curved fold lines outperform those with negatively curved fold lines. In the $x$-z plane shear loading case, although the shear stiffnesses of the folded cores with curved fold lines are lower than those of the standard Miura folded cores, the stresses and absorbed energies of the folded cores with curved fold lines with larger absolute value of $\gamma$ exceed those of the standard Miura folded cores in the post-buckling range. In both bending cases, only the folded cores with positively curved fold lines show better performances than the standard Miura folded cores.

The mechanical performances of the two-layered and three-layered folded cores are poorer than those of the single-layered model with curved folded lines in compressive and shear whereas the multiple-layered models show apparent improvement in the bending cases.

When compared with the honeycomb core with the same density and height, the folded cores show comparable or even better mechanical properties in shear and bending cases but relatively lower performance in compression.

The virtual tests reported in this paper provide an insight into the mechanical properties of various Miura-based folded cores. However, it should be noted that there are several limitations. First, the number of unit cells contained in the simulated folded core models is small, i.e. 4 or 8 due to computational time consideration. In reality, a folded core usually contains a large number of unit cells. Second, no imperfections are introduced into the numerical models while in reality there are always imperfections introduced from the manufacturing process. Third, the material model considered is a highly simplified one. Fourth, the bonds between the core and the faces and between different layers of the multiple-layered models are considered to be perfect while in reality failure of the bond may occur. Nevertheless, the virtual test results can still serve as a useful guideline for researchers and/or engineers to select the suitable folded core design for a certain application. In our ongoing work, virtual tests of various Miura-based folded cores subject to dynamic loads including low and high impacts are considered. The results will be reported in the subsequent paper. 


\section{REFERENCE}

[1] Herrmann, S.A., Zahlen, C.P. and Zuardy, I., 2005, "Sandwich Structures Technology in Commercial Aviation: Present Application and Future Trends," Sandwich Structures 7: Advancing with Sandwich Structures and Materials, Springer, pp. 13-26.

[2] CELPACT, 2009, Publishable Final Report, available at http://www.transport-research.info.

[3] Kehrle, R. and Kolax, M., 2006, "Sandwich Structures for Advanced Next Generation Fuselage Concepts," Proceedings of SAMPE Europe Technical Conference, Toulouse, France, pp. $11-16$

[4] Klett, Y. and Drechsler, K., 2011, “Designing Technical Tessellations,” Origami ${ }^{5}$ : Proceedings of Fifth International Meeting of Origami Science, Mathematics, and Education, Taylor \& Francis Group, pp. 305-322.

[5] Heimbs, S., 2009, "Virtual Testing of Sandwich Core Structures using Dynamic Finite Element Simulations," Computational Materials Science, 45(2), pp. 205-216.

[6] Heimbs, S., Middendorf, P., Kilchert, S., Johnson, A.F. and Maier, M., 2007, “Experimental and Numerical Analysis of Composite Folded Sandwich Core Structures under Compression," Applied Composite Materials, 14(5-6), pp. 363-377.

[7] Heimbs, S., Mehrens, T., Middendorf, P., Maier, M. and Schumacher, A., 2007, "Numerical Determination of the Nonlinear Effective Mechanical Properties of Folded Core Structures for Aircraft Sandwich Panels," Proceedings of $6^{\text {th }}$ European LS-Dyna Users Conference, Gothenburg, Sweden.

[8] Heimbs, S., Cichosz, J., Kilchert, S. and Klaus, M., 2009, "Sandwich Panels with Cellular Cores Made of Folded Composite Material: Mechanical Behaviour and Impact Performance," Proceedings of $17^{\text {th }}$ International Conference on Composite Materials, Edinburgh, United Kingdom.

[9] Baranger, E., Cluzel, C. and Guidault, P.A., 2011, "Modelling of the Behaviour of Aramid Folded Cores Up to Global Crushing,” Strain, 47(2), pp. 170-178.

[10] Heimbs, S., Cichosz, J., Klaus, M., Kilchert, S. and Johnson, A.F., 2010, "Sandwich Structures with Textile-Reinforced Composite Foldcores under Impact Loads," Composite Structures, 92(6), pp. 1485-1497. 
[11] Heimbs, S., Kilchert, S., Fischer, S., Klaus, M. and Baranger, E., 2009, "Sandwich Structures with Folded Core: Mechanical Modelling and Impact Simulations," Proceedings of SAMPE Europe International Conference, Paris, France.

[12] Nguyen, M.Q., Jacombs, S.S., Thomson, R.S., Hachenberg, D. and Scott, M.L., 2005, “Simulation of Impact on Sandwich Structures," Composite Structures, 67(2), 217-227.

[13] Klaus, M. and Reimderdes, H.G., 2009, "Residual Strength Simulations of Sandwich Panels after Impact," Proceedings of $17^{\text {th }}$ International Conference on Composite Materials, Edinburgh, United Kingdom.

[14]Heimbs, S., 2013, "Foldcore Sandwich Structures and Their Impact Behaviour: An Overview," Solid Mechanics and Its Applications, 192, pp. 491-544. (DOI: 10.1007/978-94-007-5329-7_11)

[15] Miur, k., 1975, "New Structural Form of Sandwich Core," Journal of Aircraft, 12(5), pp. 437-441. DOI: $10.2514 / 3.44468$

[16] Zhou, X., Wang, H. and You, Z., 2014, “A Method of Designing Developable Origami Structures in Three-Dimensional Space - Part A: Mathematical Foundation," Proceedings of the ASME 2014 International Design Engineering Technical Conferences \& Computers and Information in Engineering Conference, Buffalo, New York, USA.

[17] Hou, S., Zhao, S., Ren, L., Han, X. and Li, Q., 2013, "Crashworthiness Optimization of Corrugated Sandwich Panels," Materials and Design, 51, pp. 1071-1084. 


\section{CAPTIONS OF FIGURES}

Fig. 1 The input points in the $x-z$ and $y-z$ planes used to generate the unit cell models UM11-UM18.

Fig. 2 Unit cell models UM11-UM18.

Fig. 3 Definition of the base area $S_{u}$ and the core height $H_{c}$ of a unit cell.

Fig. 4 Folded core models M11-M18 each consisting of four unit cells in a $2 \times 2$ array.

Fig. 5 (a) Virtual compression test; (b) virtual shear test in the $y$-z plane; (c) virtual shear test in the $x$-z plane.

Fig. $6 \quad$ Virtual bending test (a) in the $x$-direction and (b) in the $y$-direction.

Fig. 7 The effective compressive stress versus strain curves of models M11-M18 in the virtual compression test.

Fig. 8 The deformed shapes of model M11 in the virtual compression test at: (a) $2 \%$ strain; (b) $20 \%$ strain; (c) $50 \%$ strain.

Fig. 9 The absorbed compressive energy per area versus strain curves of models M11M18 in the virtual compression test.

Fig. 10 The effective shear stress versus strain curves of models M11-M18 in the virtual shear tests (a) in the $y$-z plane and (b) in the $x$-z plane.

Fig. 11 The deformed shapes of model M11 in the virtual shear test in the $y$-z plane at: (a) $2 \%$ strain; (b) $20 \%$ strain; (c) $50 \%$ strain.

Fig. 12 The absorbed shear energy per area versus strain curves of models M11-M18 in the virtual shear tests (a) in the $y$-z plane and (b) in the $x$-z plane.

Fig. 13 (a) The bending moment normalized by $l_{x}$ versus curvature curves of models M11-M18 in the virtual bending test in the $x$-direction; (b) the bending moment normalized by $l_{y}$ versus curvature curves of models M11 to M18 in the virtual bending test in the $y$-direction.

Fig. 14 The deformed shapes of model M11 in the virtual bending tests (a) in the $x$-direction and (b) in the $y$-direction.

Fig. 15 The $x$-z plane inputs used to generate the unit cell models UM21-UM28. 
Fig. 16 Folded core models M21-M28 each consisting of four unit cells in a $2 \times 2$ array.

Fig. 17 The effective compressive stress versus strain curves of models M21-M28 and M11 in the virtual compression test.

Fig. 18 The absorbed compressive energy per area versus strain curves of models M21-M28 and M11 in the virtual compression test.

Fig. 19 The effective shear stress versus strain curves of models M21-M28 and M11 in the virtual shear tests (a) in the $y$-z plane and (b) in the $x-z$ plane.

Fig. 20 The absorbed shear energy per area versus strain curves of models M21-M28 and M11 in the virtual shear tests (a) in the $y$-z plane and (b) in the $x$-z plane.

Fig. 21 (a) The bending moment normalized by $l_{x}$ versus curvature curves of models M21-M28 and M11 in the virtual bending test in the $x$-direction; (b) the bending moment normalized by $l_{y}$ versus curvature curves of models M21-M28 and M11 in the virtual bending test in the $y$-direction.

Fig. 22 Folded core models M31 and M32 that consist of two- and three-layers, respectively.

Fig. 23 The effective compressive stress versus strain curves of models M31, M32, M21, M11 and HMC in the virtual compression test.

Fig. 24 The effective shear stress versus strain curves of models M31, M32, M21, M11 and HMC in the virtual shear tests (a) in the $y$-z plane and (b) in the $x$-z plane.

Fig. 25 (a) The bending moment normalized by $l_{x}$ versus curvature curves of models M31, M32, M21, M11 and HMC in the virtual bending test in the $x$-direction; (b) the bending moment normalized by $l_{y}$ versus curvature curves of models M31, M32, M21, M11 and HMC in the virtual bending test in the $y$-direction.

Fig. 26 (a) The honeycomb core unit cell geometries; (b) honeycomb core model MHC consisting of four unit cells in a $2 \times 2$ array. 


\section{CAPTIONS OF TABLES}

Table 1 The geometric properties of models M11-M18.

Table 2 The parameters of the material model.

Table 3 The compressive stiffness $E_{c}$ and shear stiffnesses $E_{s 1}$ and $E_{s 2}$ of models M11-M18.

Table 4 The bending stiffness $E I_{x}$, maximum bending moment $M_{x}^{c r}$ and buckling curvature $k_{x}^{c r}$ in the $x$-direction and the bending stiffness $E I_{y}$, maximum bending moment $M_{y}^{c r}$ and buckling curvature $k_{y}^{c r}$ in the $y$-direction of models M11-M18.

Table 5 The geometric properties of models M21-M28 where $\alpha=\pi / 4, h_{x}=5 \mathrm{~mm}, \beta=$ $\pi / 4$ and $h_{y}=10 \mathrm{~mm}$

Table 6 The compressive stiffness $E_{c}$ and strength $\sigma_{c}^{c r}$, shear stiffnesses $E_{s 1}$ and $E_{s 2}$ and strengths $\sigma_{s 1}^{c r}$ and $\sigma_{s 2}^{c r}$ of models M21-M28.

Table 7 The bending stiffness $E I_{x}$, maximum bending moment $M_{x}^{c r}$ and buckling curvature $k_{x}^{c r}$ in the $x$-direction and the bending stiffness $E I_{y}$, maximum bending moment $M_{y}^{c r}$ and buckling curvature $k_{y}^{c r}$ in the $y$-direction of models M21-M28.

Table 8 The geometric properties of models M31-M32 where $\alpha=\pi / 4, h_{x}=2.5 \mathrm{~mm}$, $\beta=\pi / 4$ and $h_{y}=5 \mathrm{~mm}$ 
Figure 1
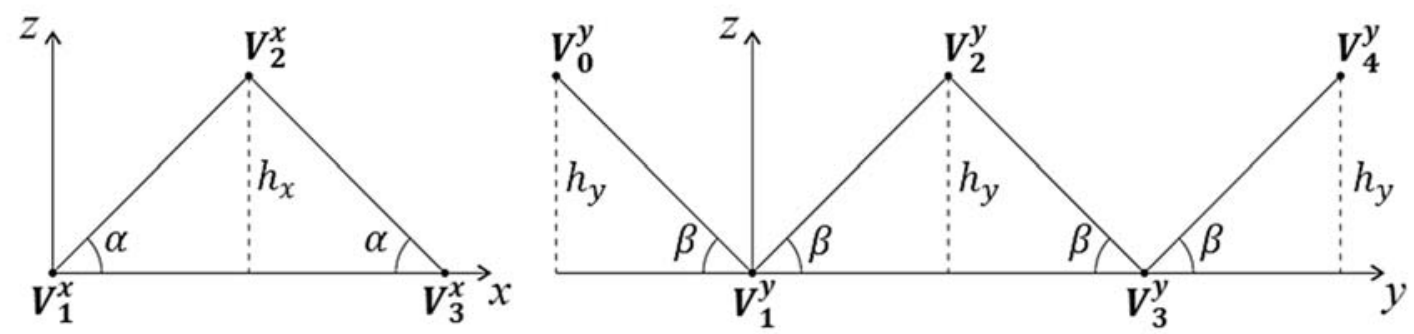
Figure 2

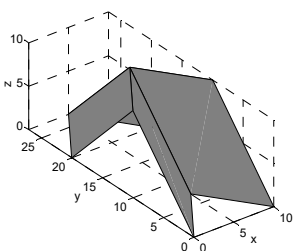

UM11

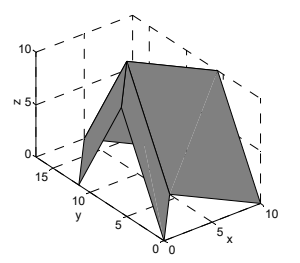

UM15

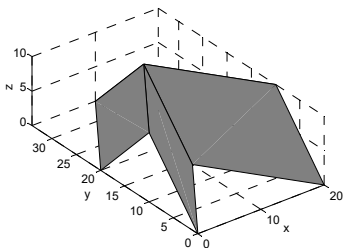

UM12

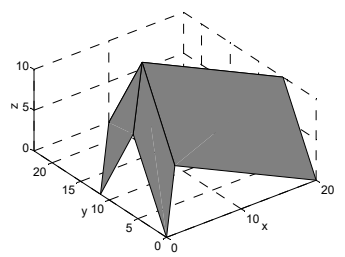

UM16

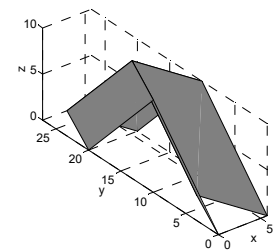

UM13

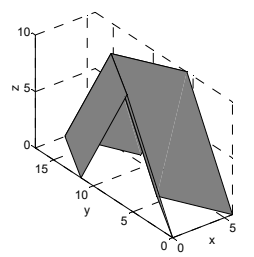

UM17

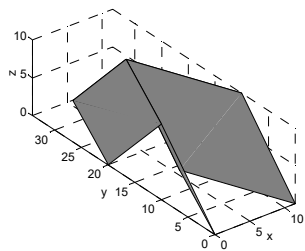

UM14

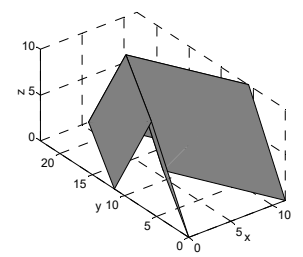

UM18 
Figure 3

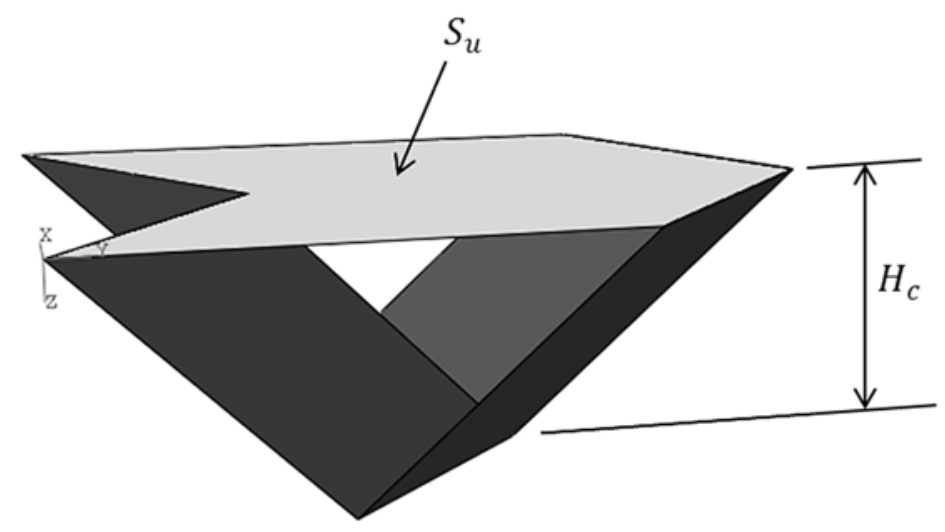


Figure 4

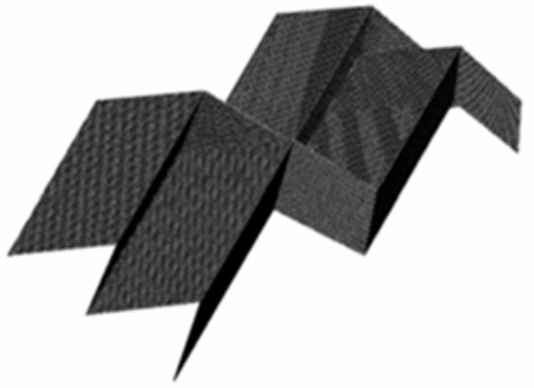

M11

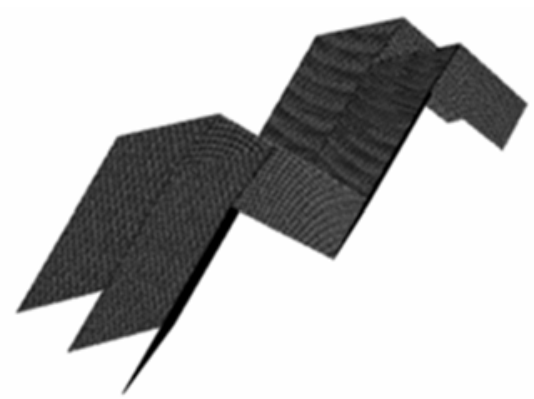

M13

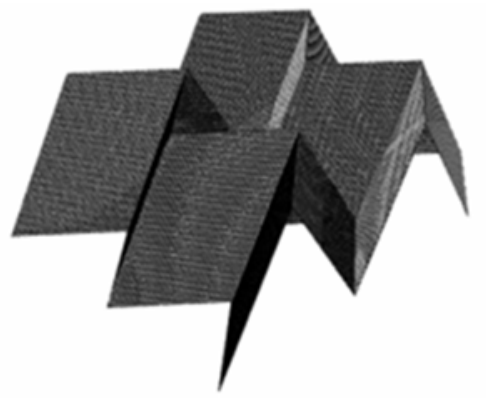

M15

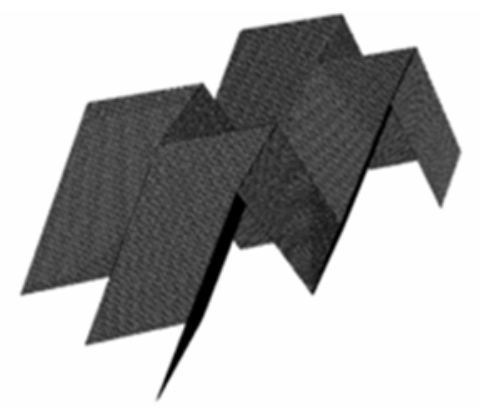

M17

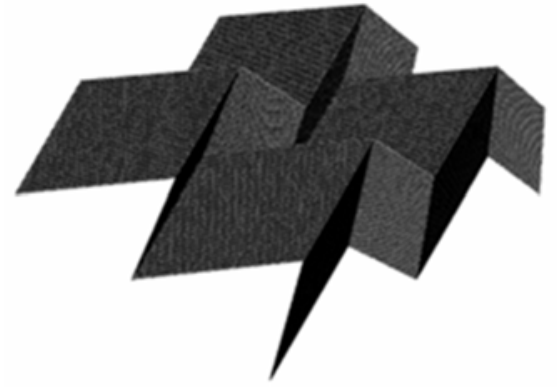

M12

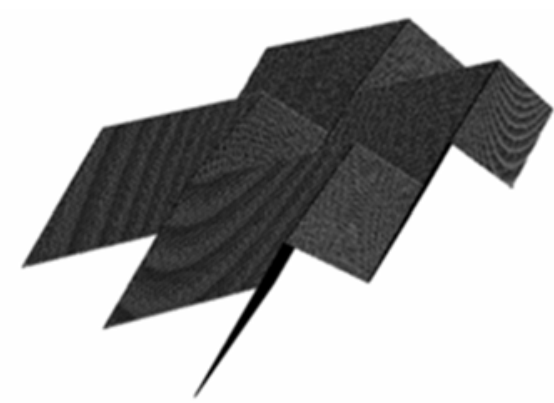

M14

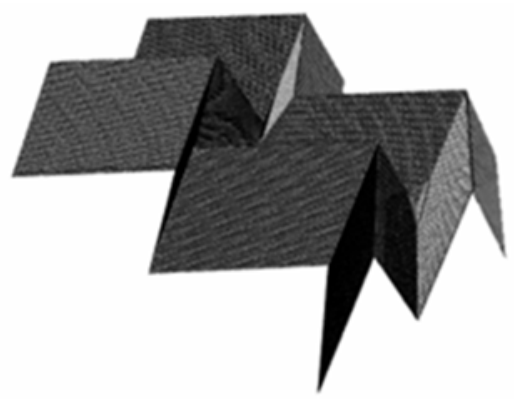

M16

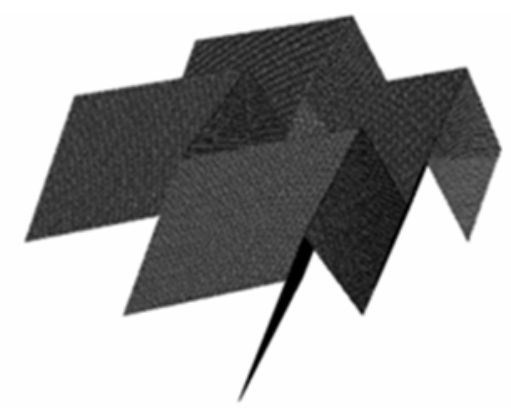

M18 
Figure 5

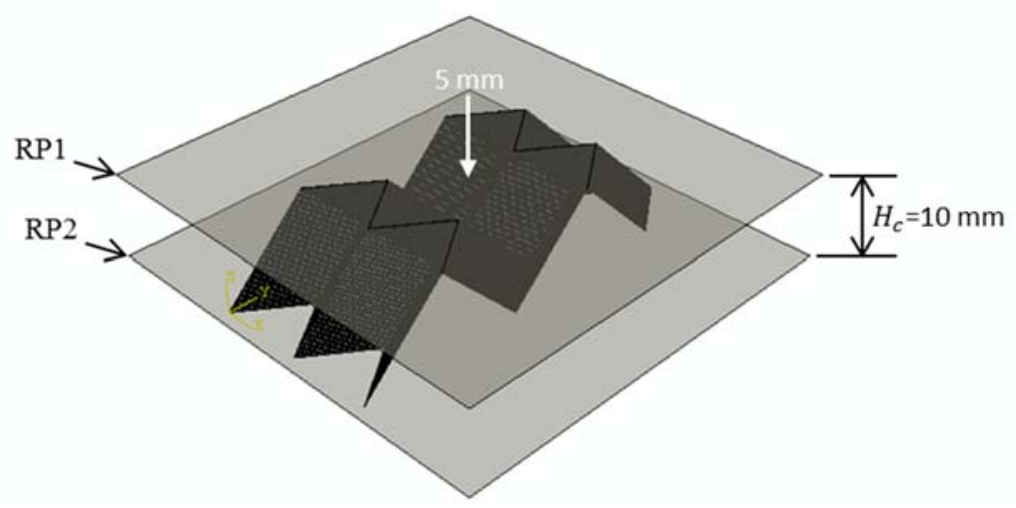

(a)

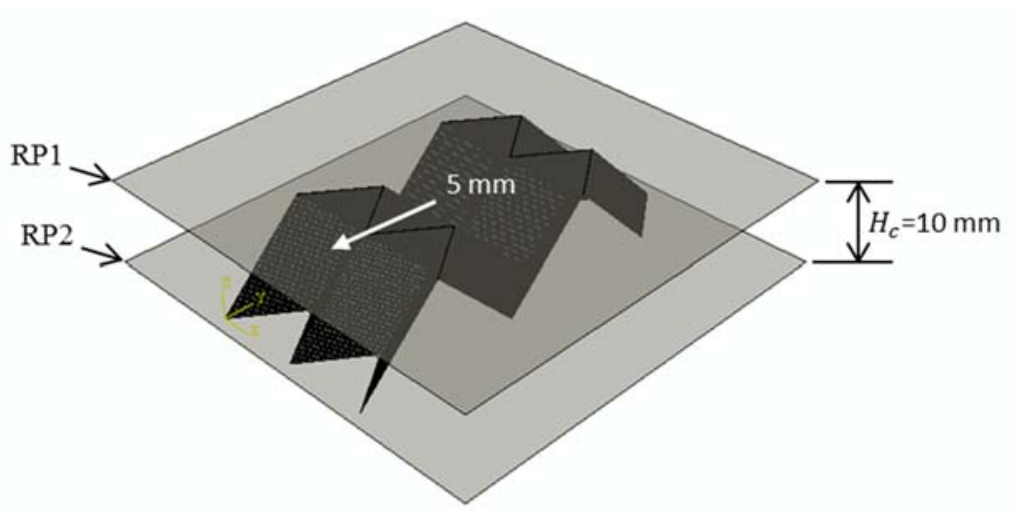

(b)

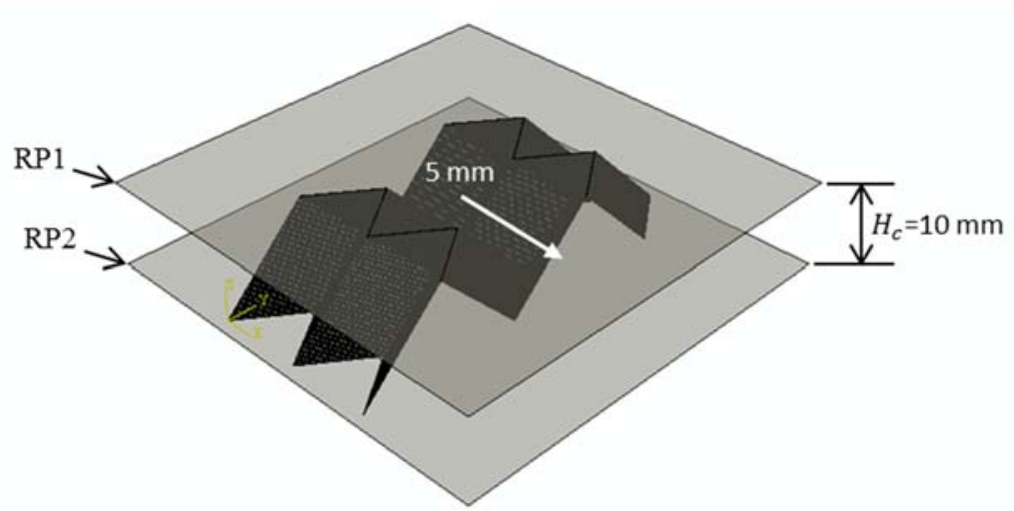

(c) 
Figure 6

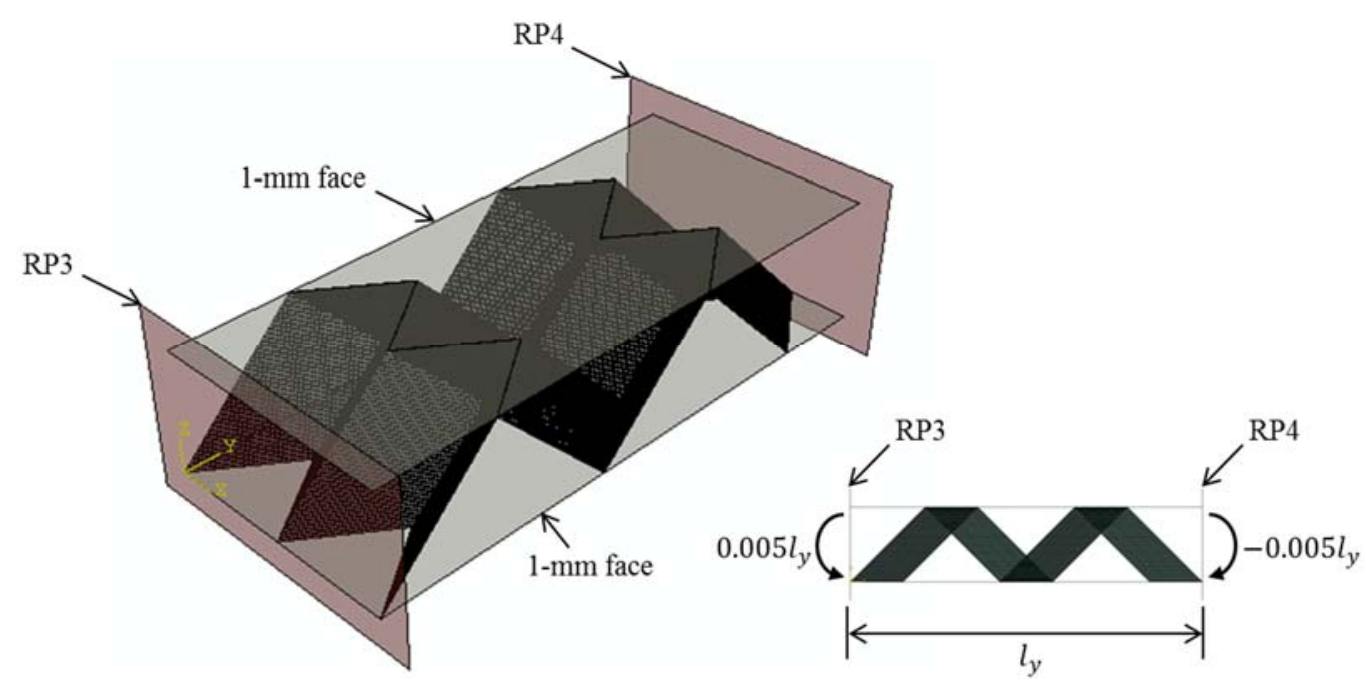

(a)

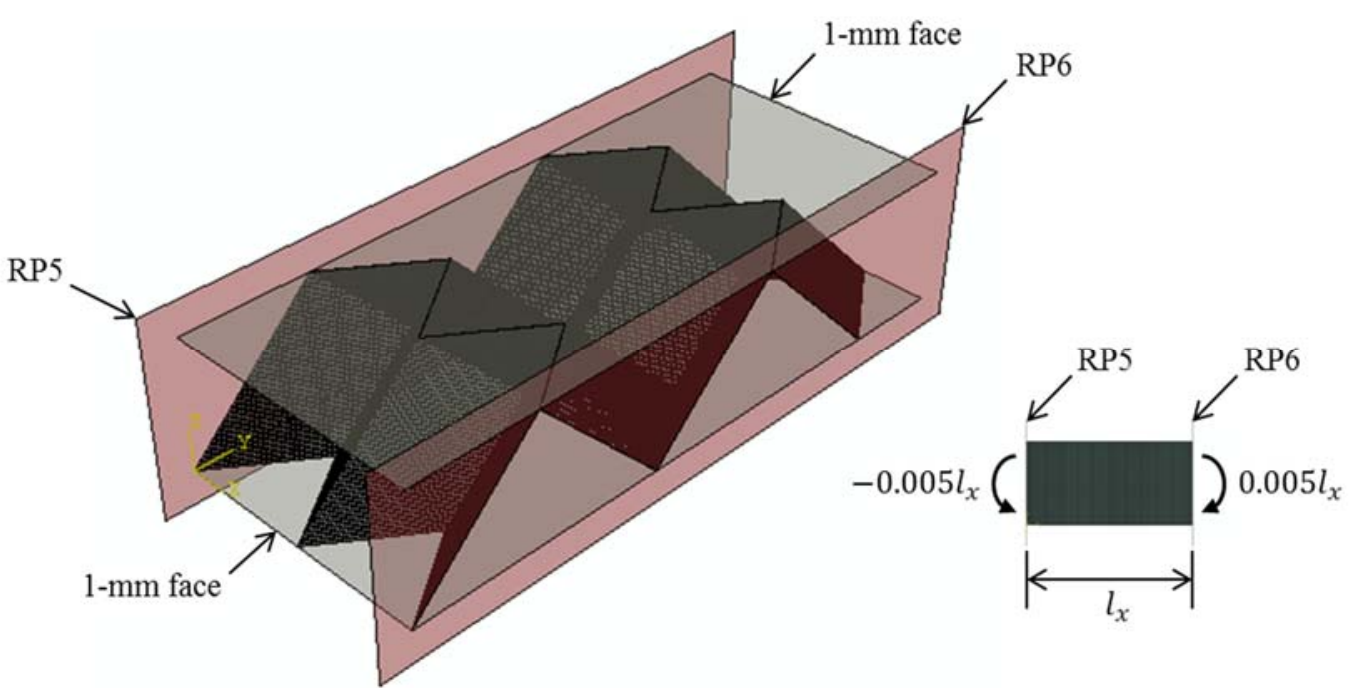

(b) 
Figure 7

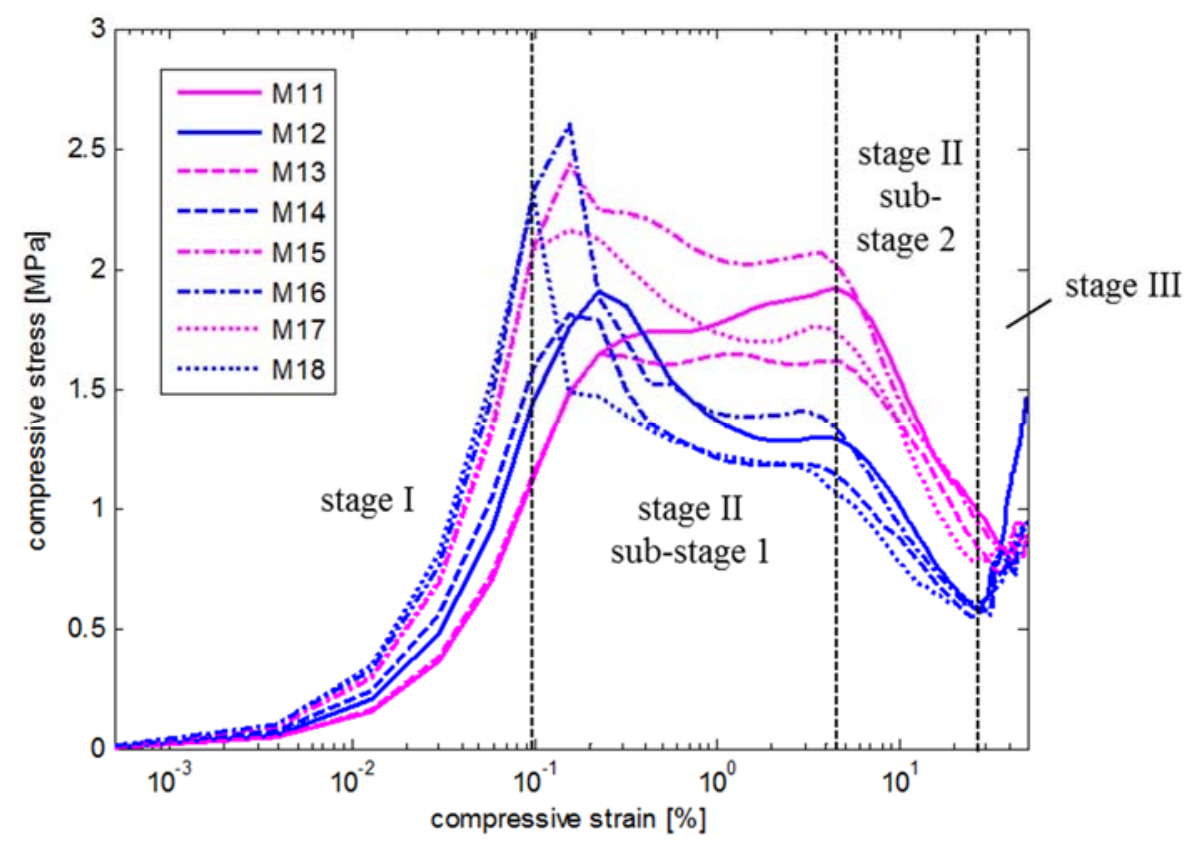


Figure 8

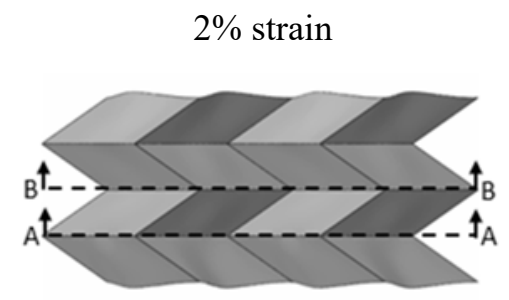

top view

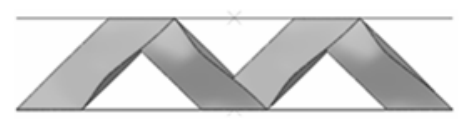

side view

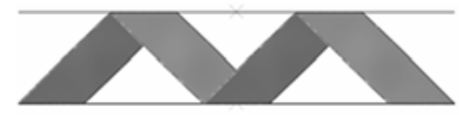

section A-A

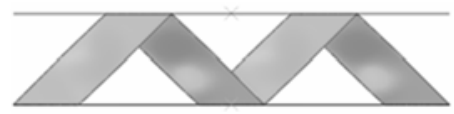

section B-B

(a)
$20 \%$ strain

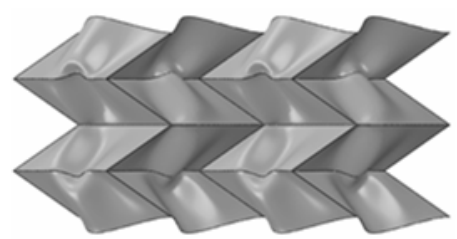

top view

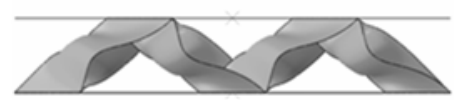

side view

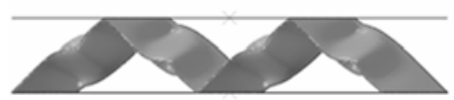

section A-A

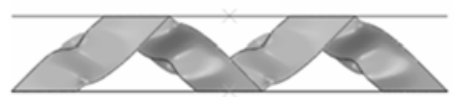

section B-B

(b)
$50 \%$ strain

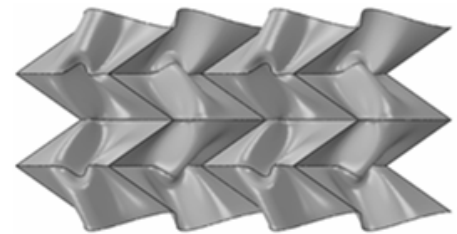

top view

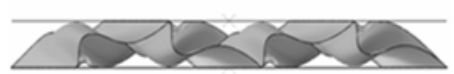

side view

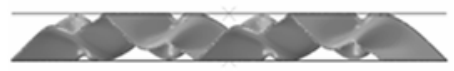

section A-A

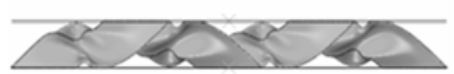

section B-B

(c) 
Figure 9

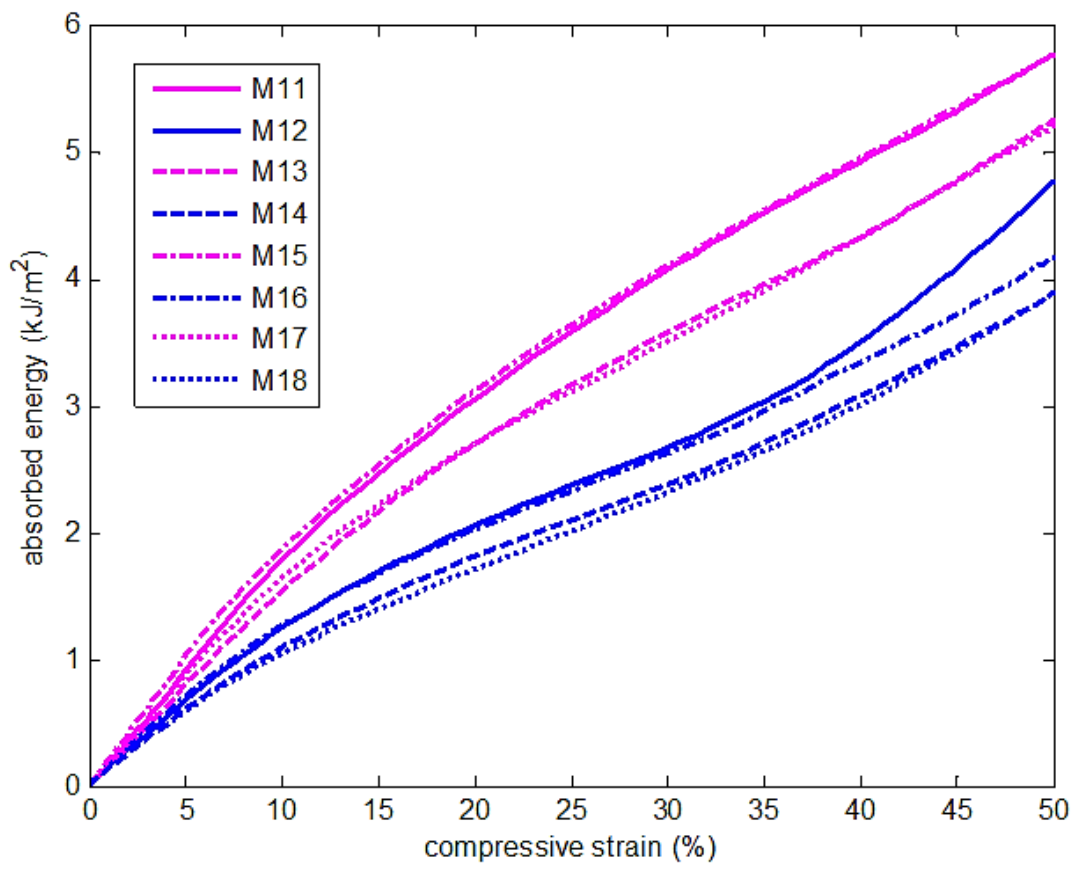


Figure 10

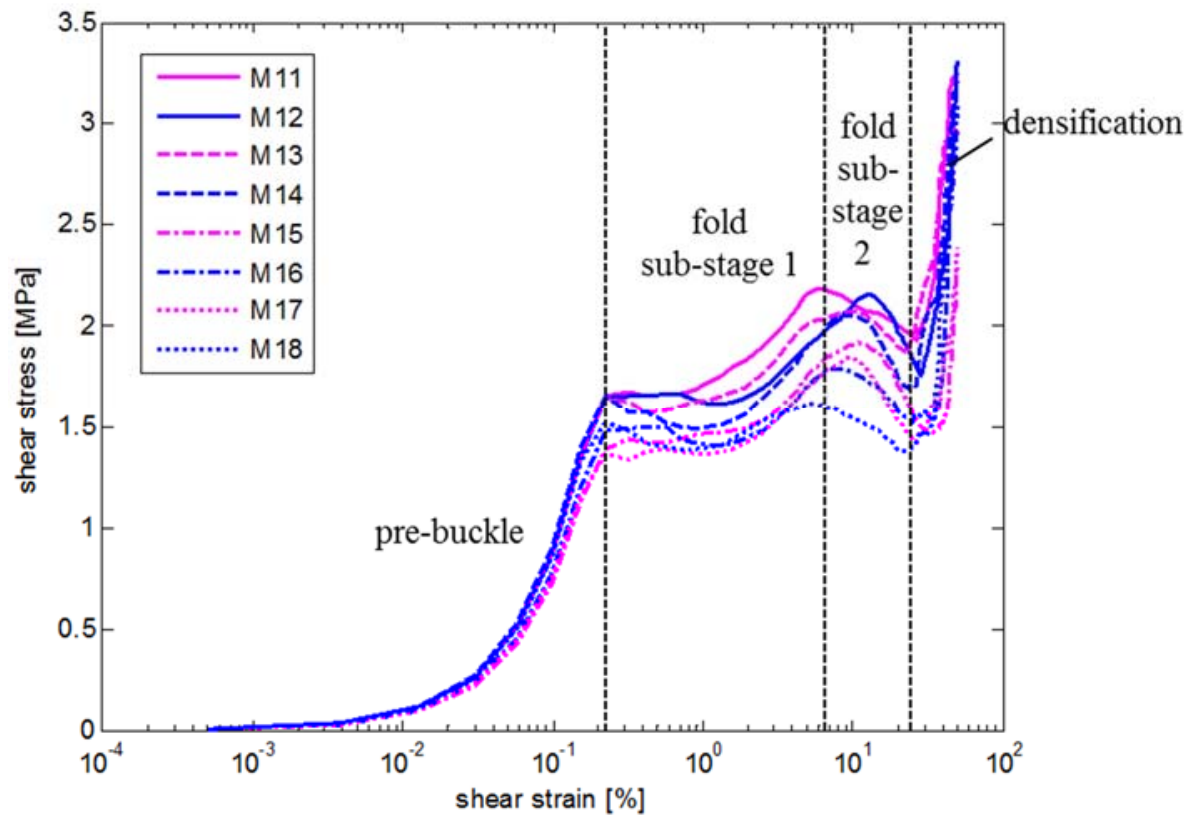

(a)

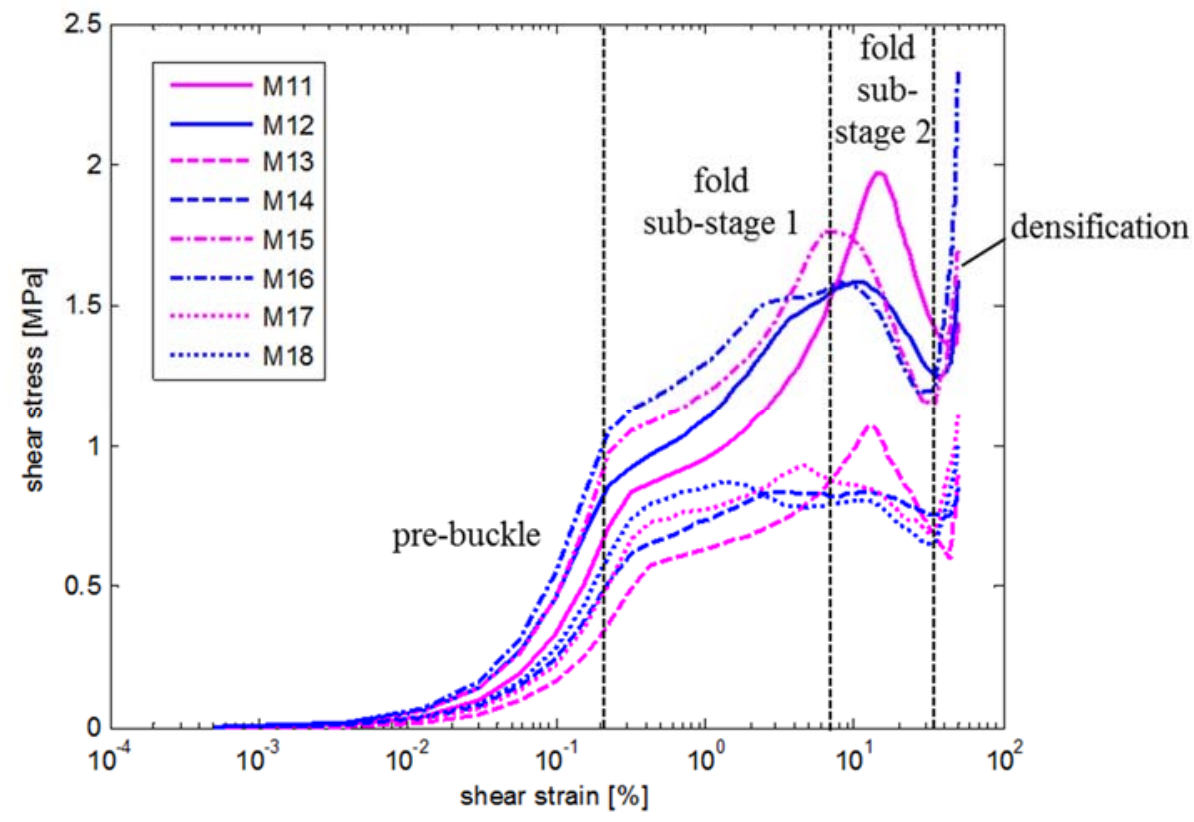

(b) 
Figure 11

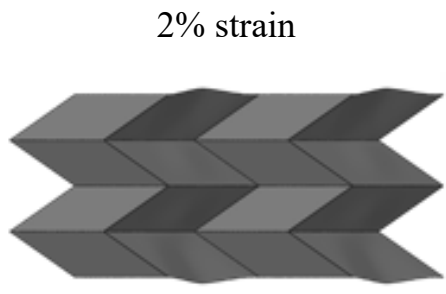

top view

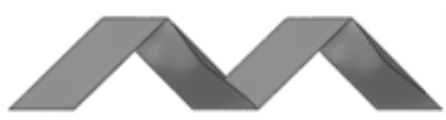

side view

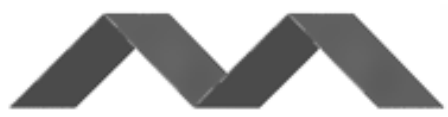

section A-A

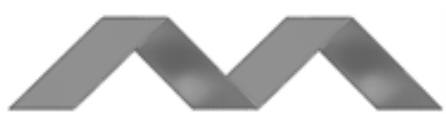

section B-B

(a)

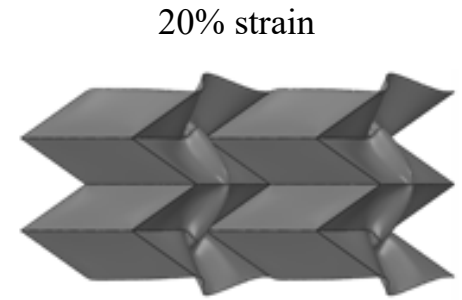

top view

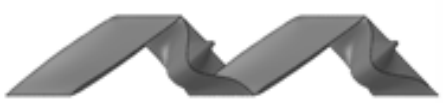

side view

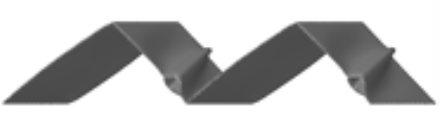

section A-A

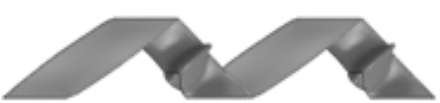

section B-B

(b)

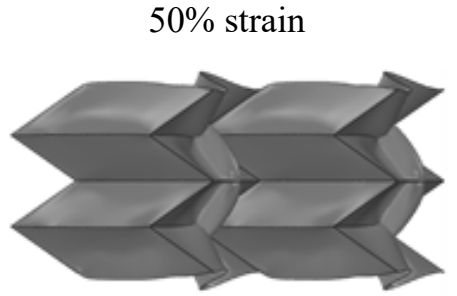

top view

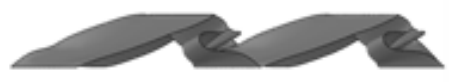

side view

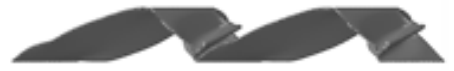

section A-A

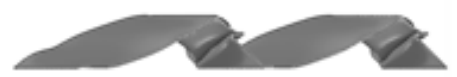

section $\mathrm{B}-\mathrm{B}$

(c) 
Figure 12

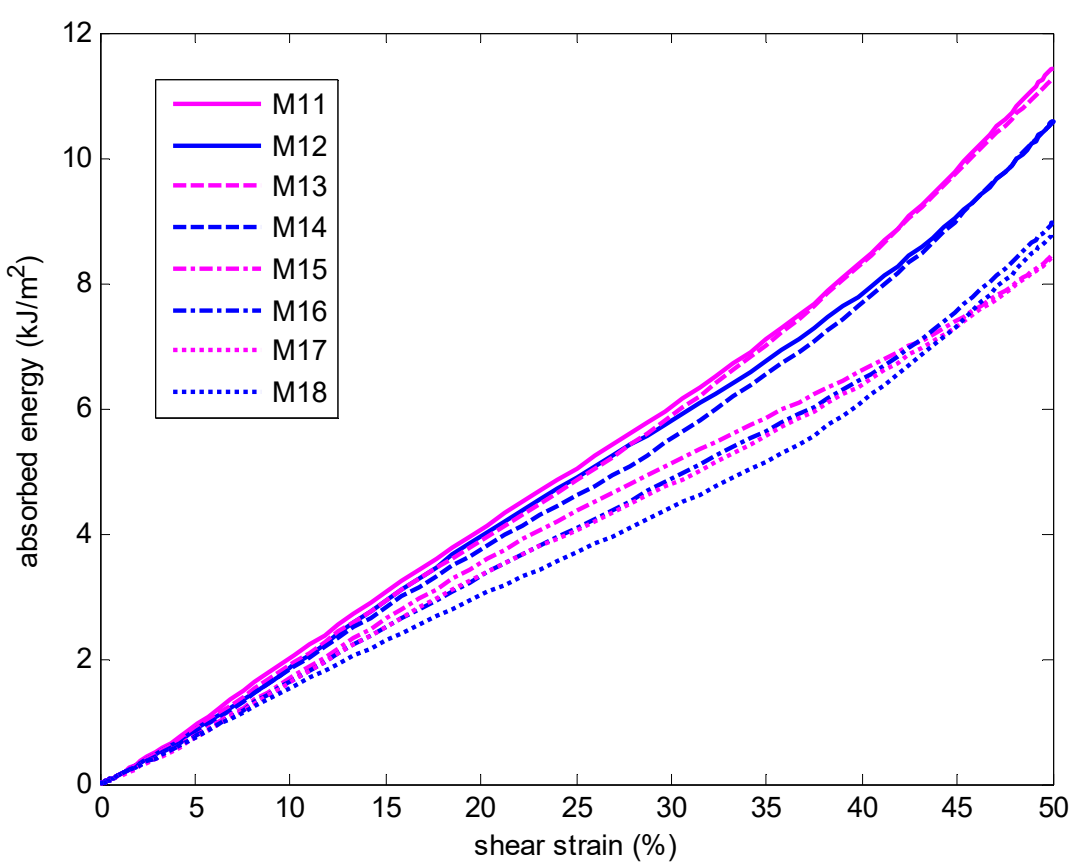

(a)

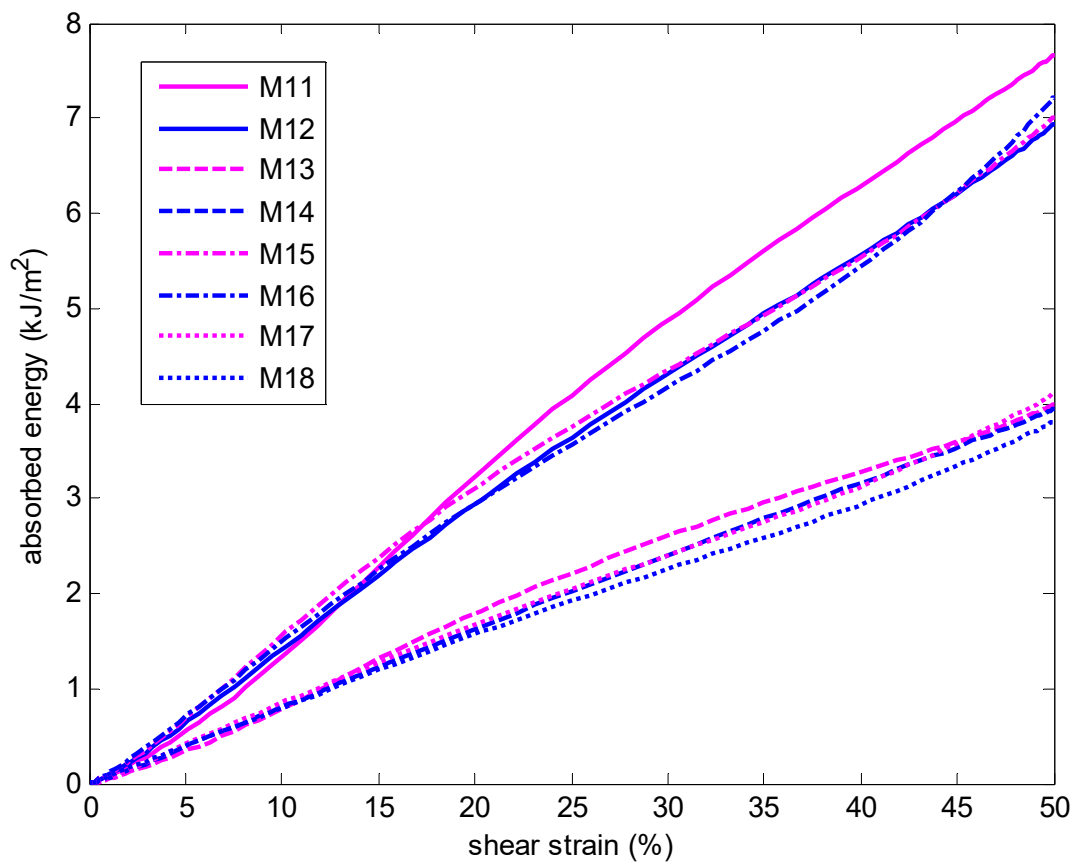

(b) 
Figure 13

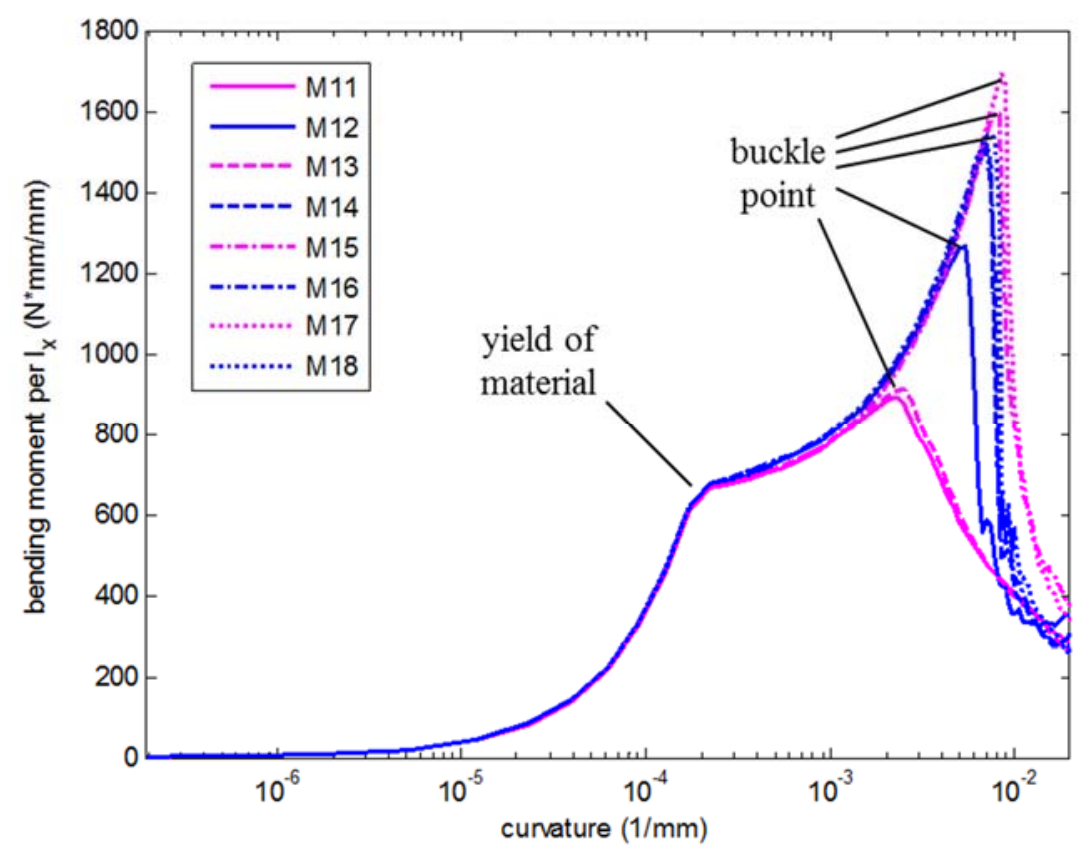

(a)

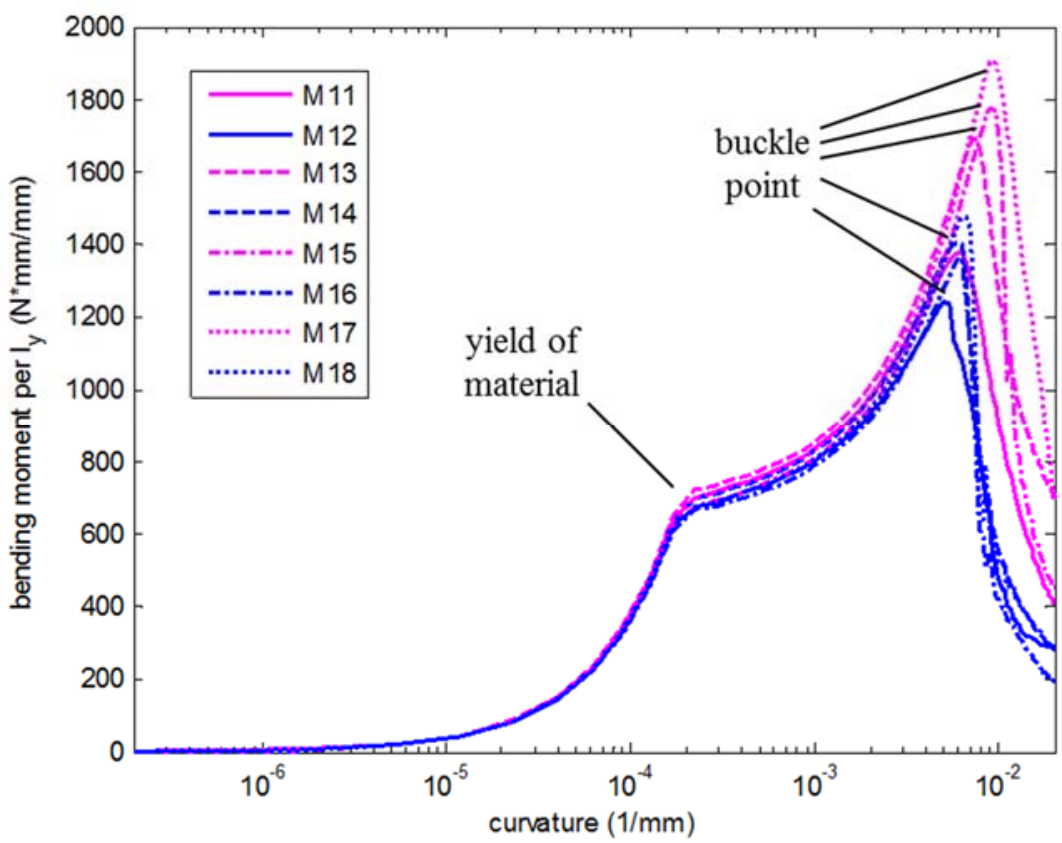

(b) 
Figure 14

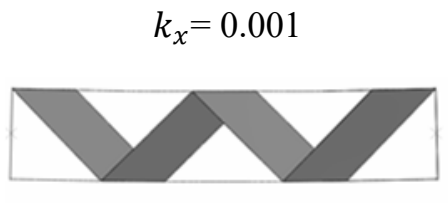

$k_{y}=0.004$

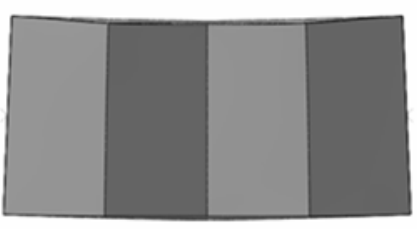

$k_{x}=0.003$

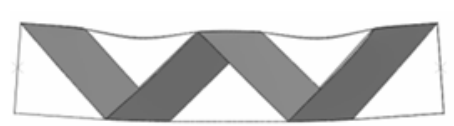

(a)

$k_{y}=0.008$

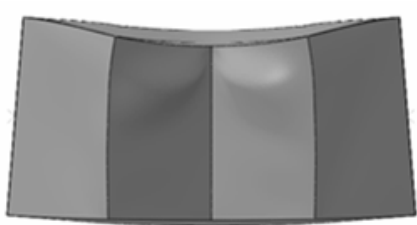

(b)

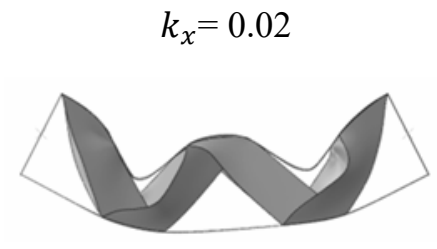

$k_{y}=0.02$

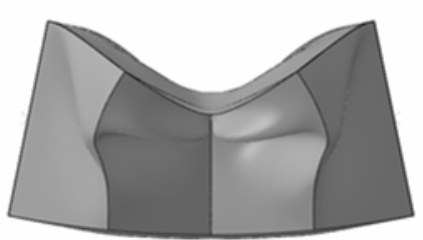


Figure 15

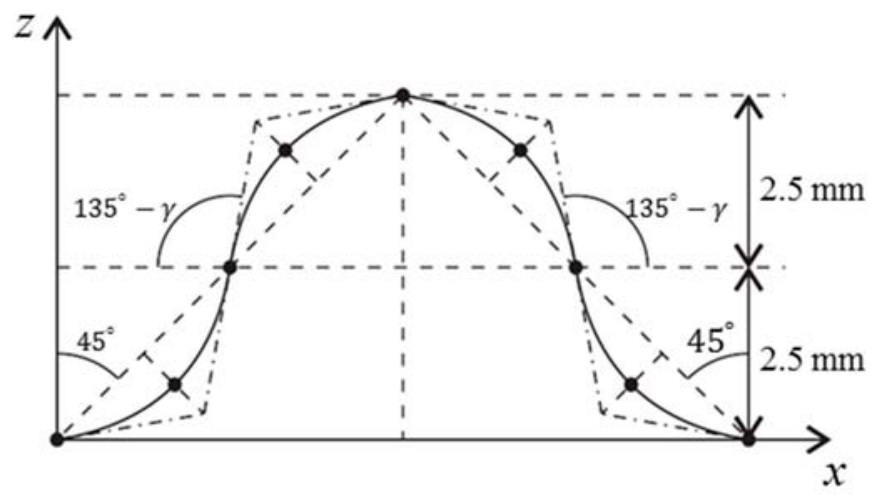


Figure 16

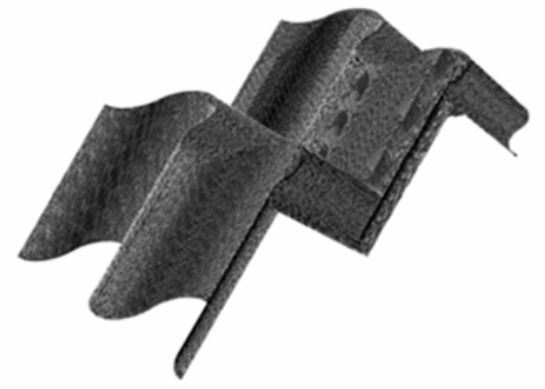

M21

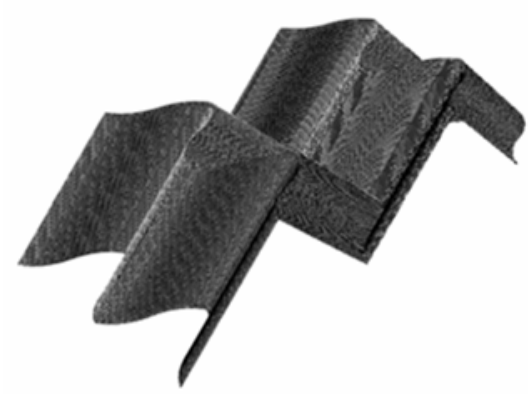

M23

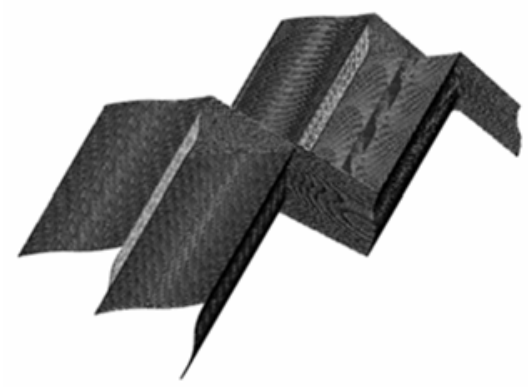

M25

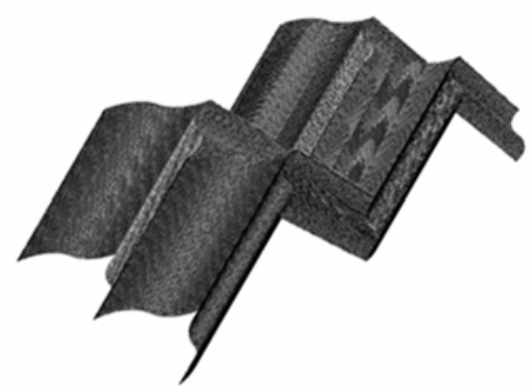

M27

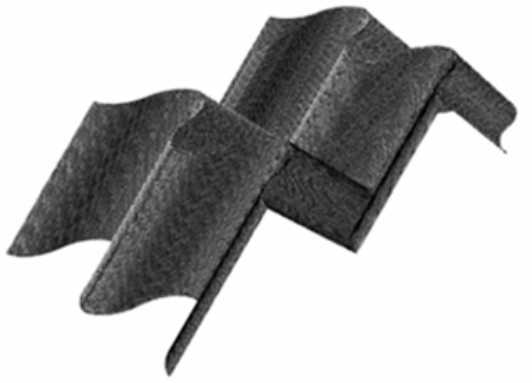

M22

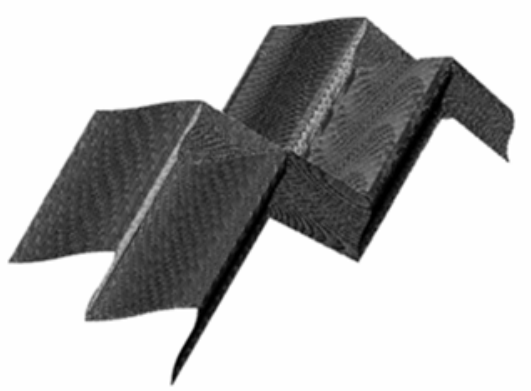

M24

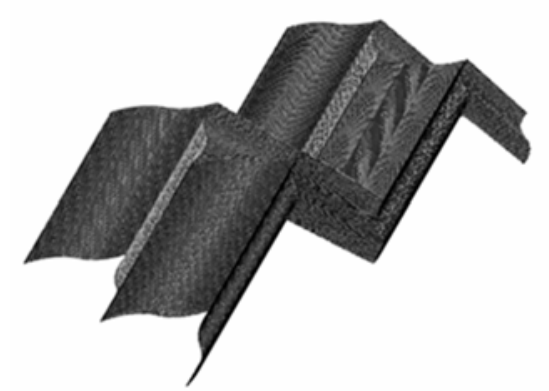

M26

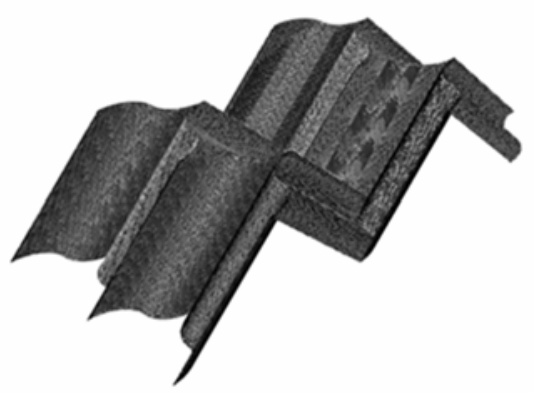

M28 
Figure 17

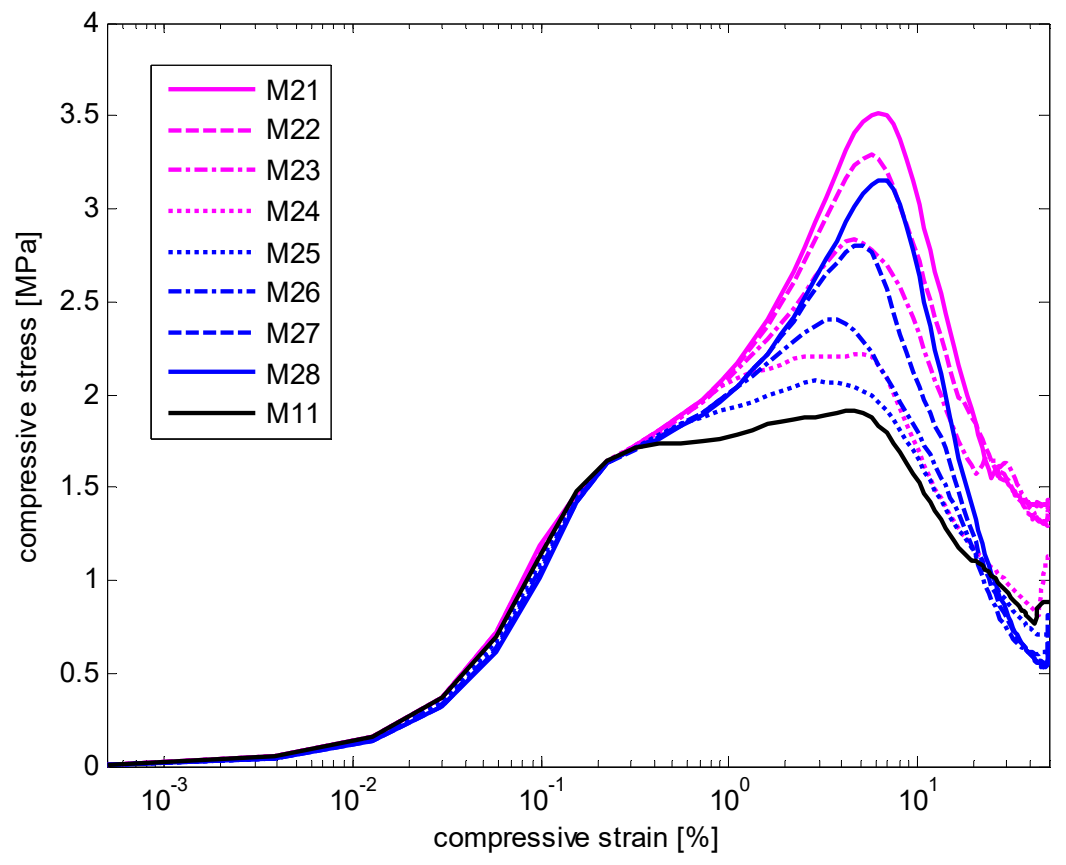


Figure 18

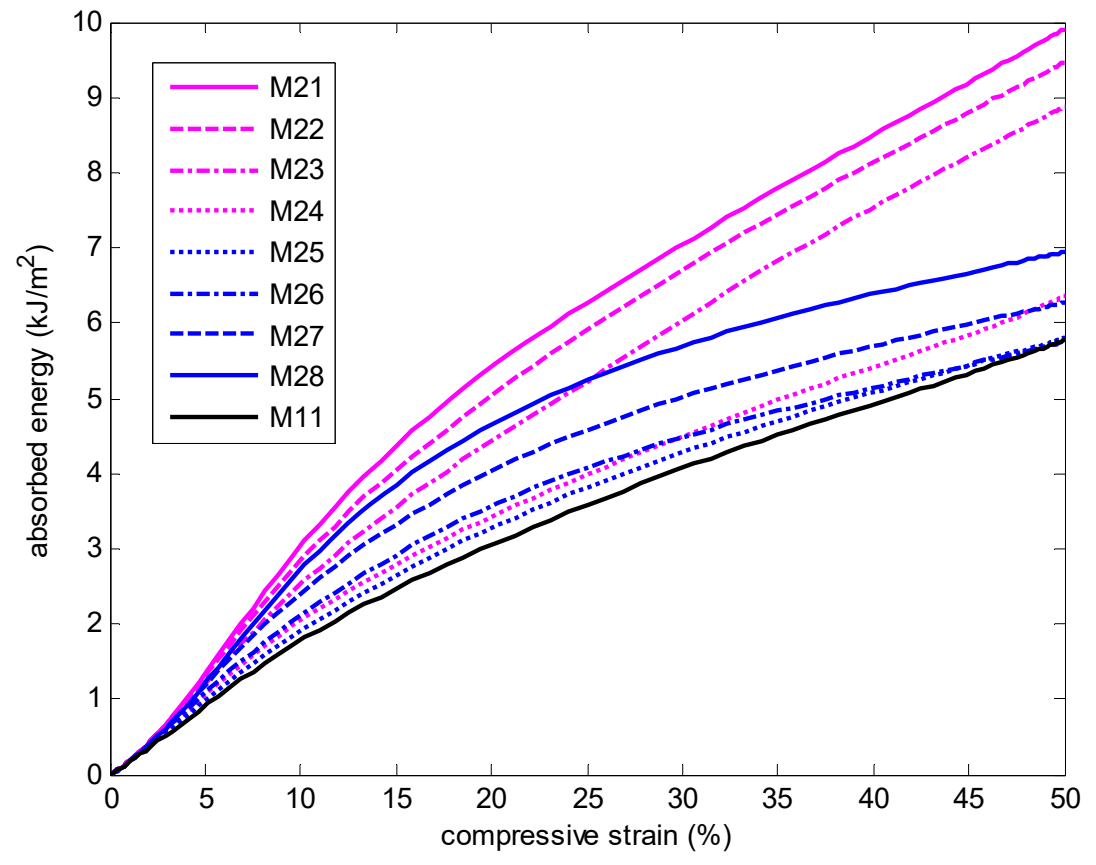


Figure 19

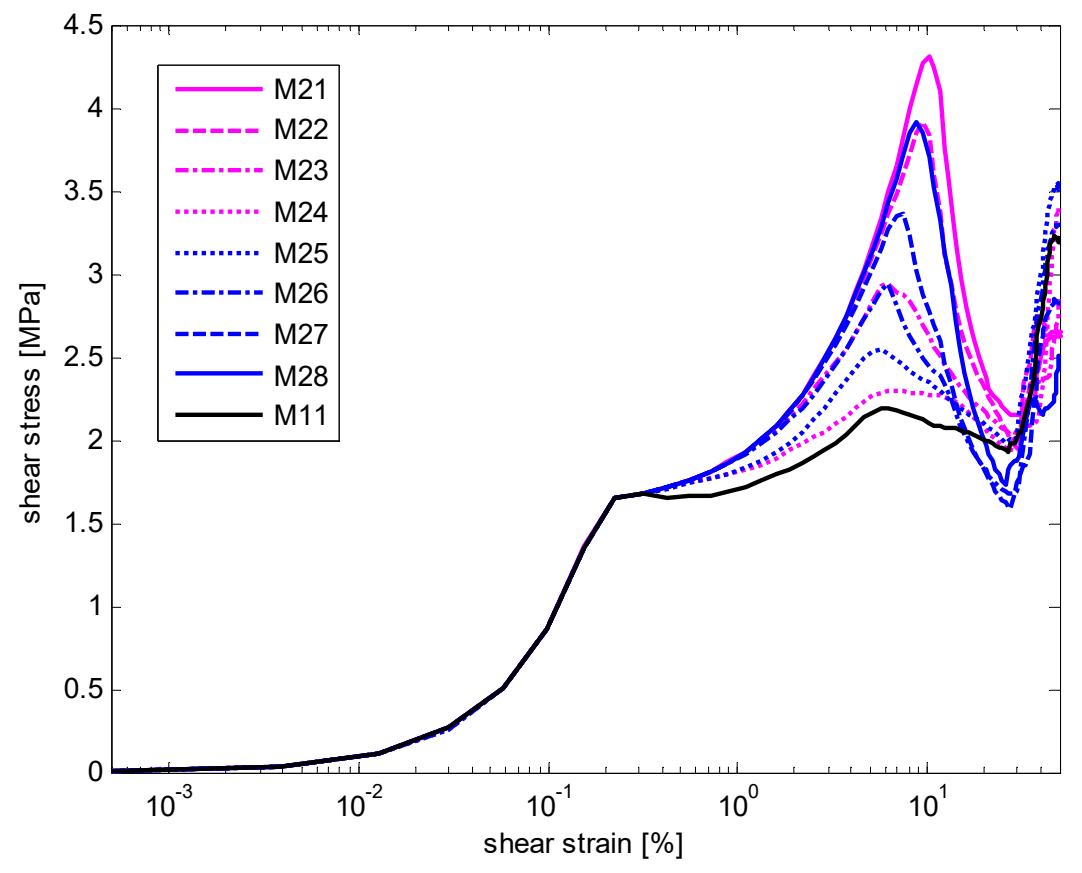

(a)

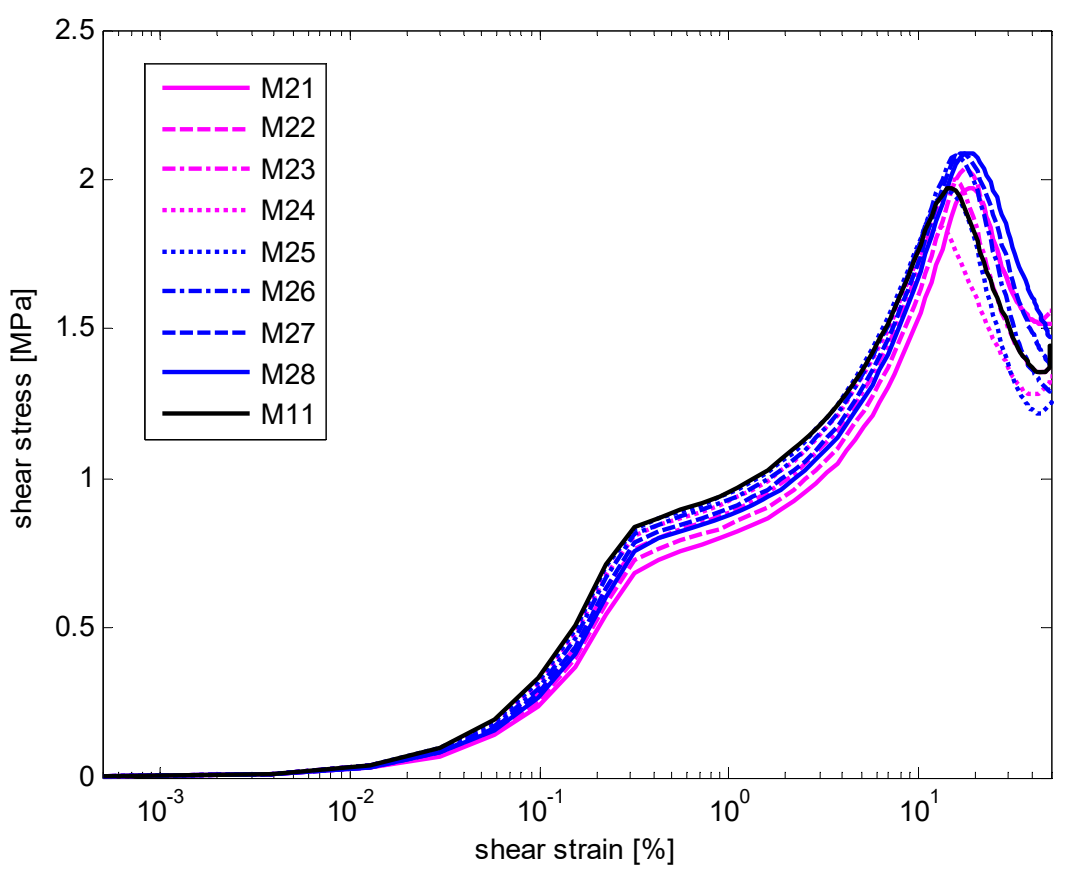

(b) 
Figure 20

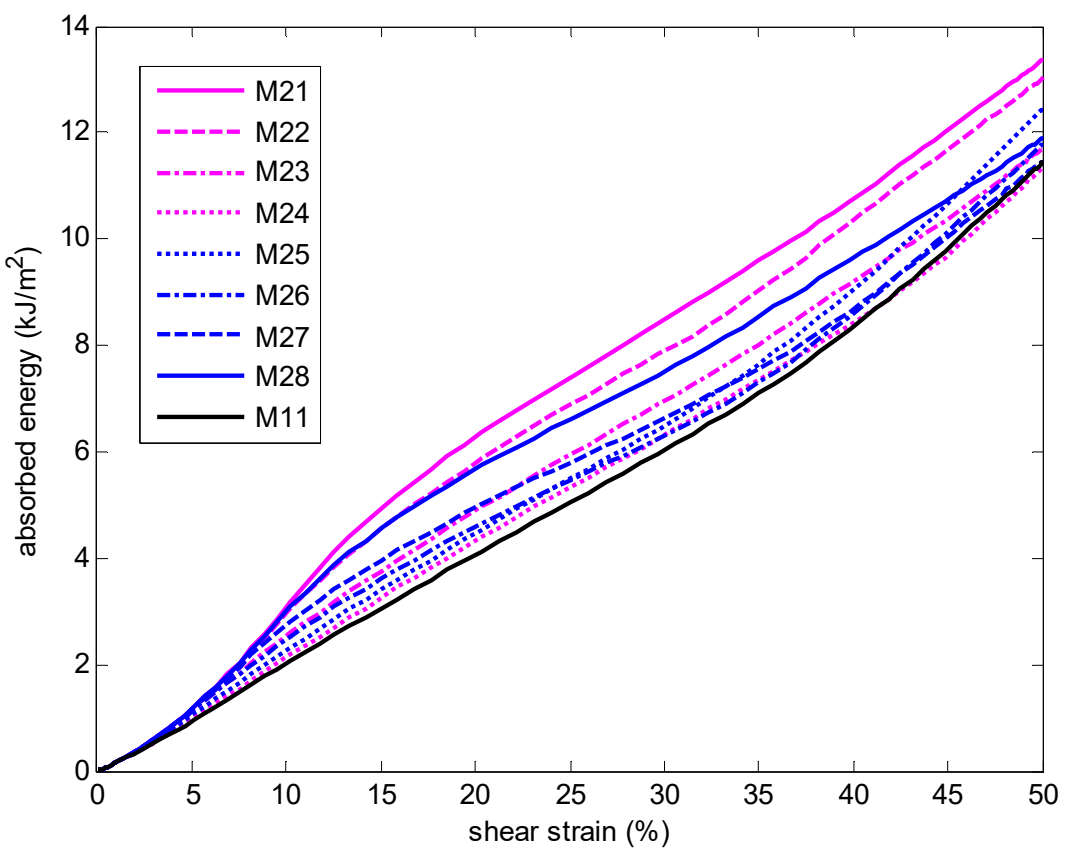

(a)

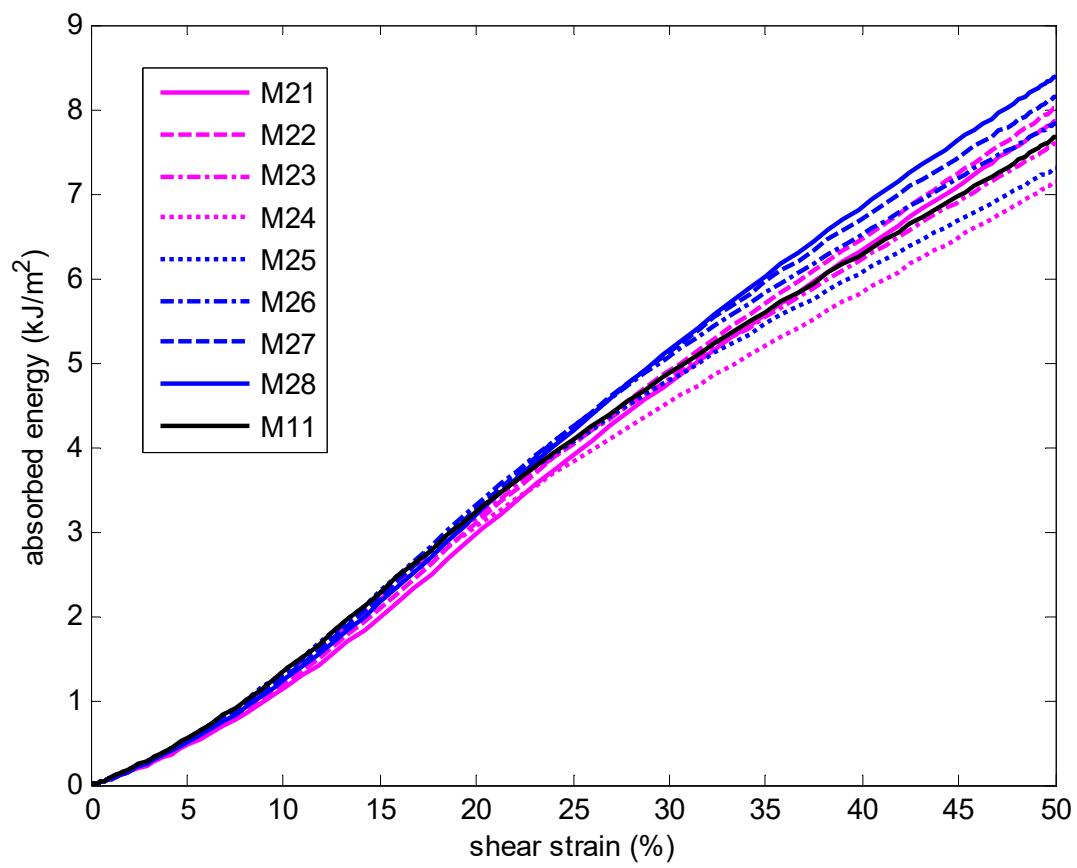

(b) 
Figure 21

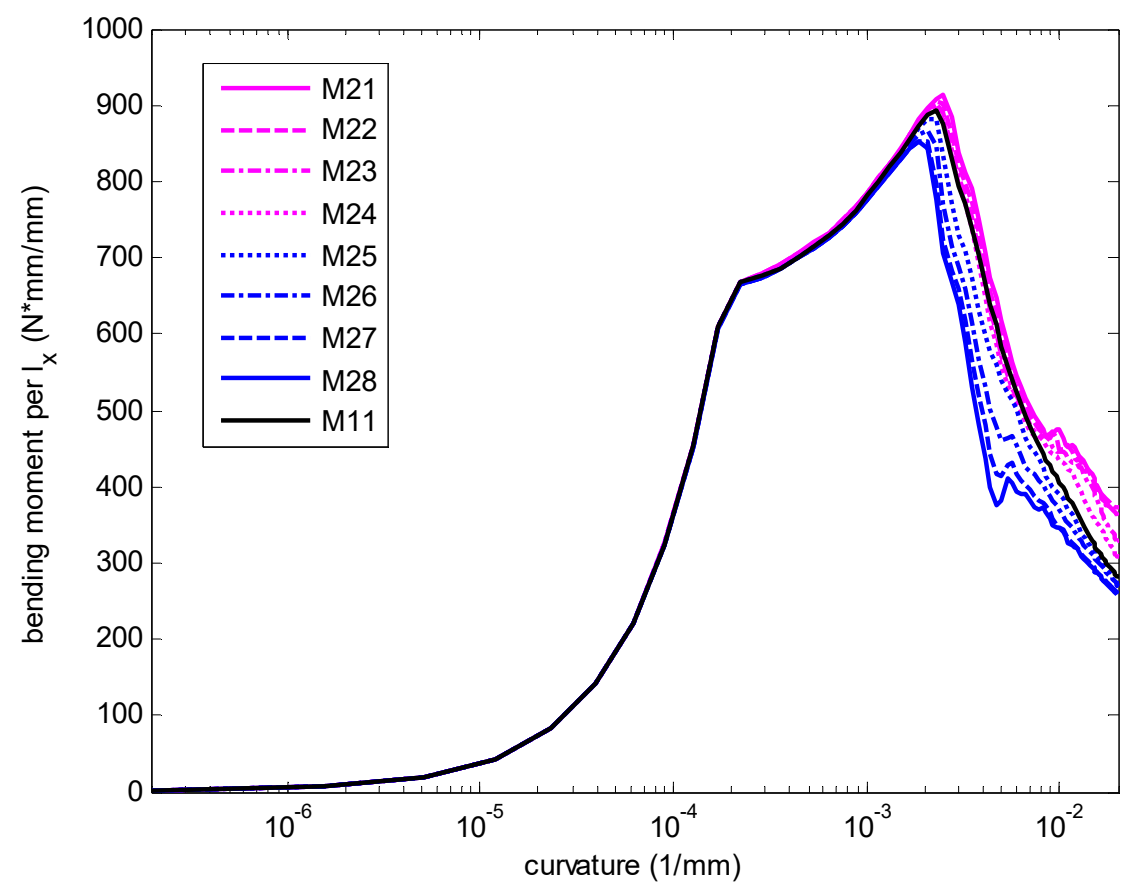

(a)

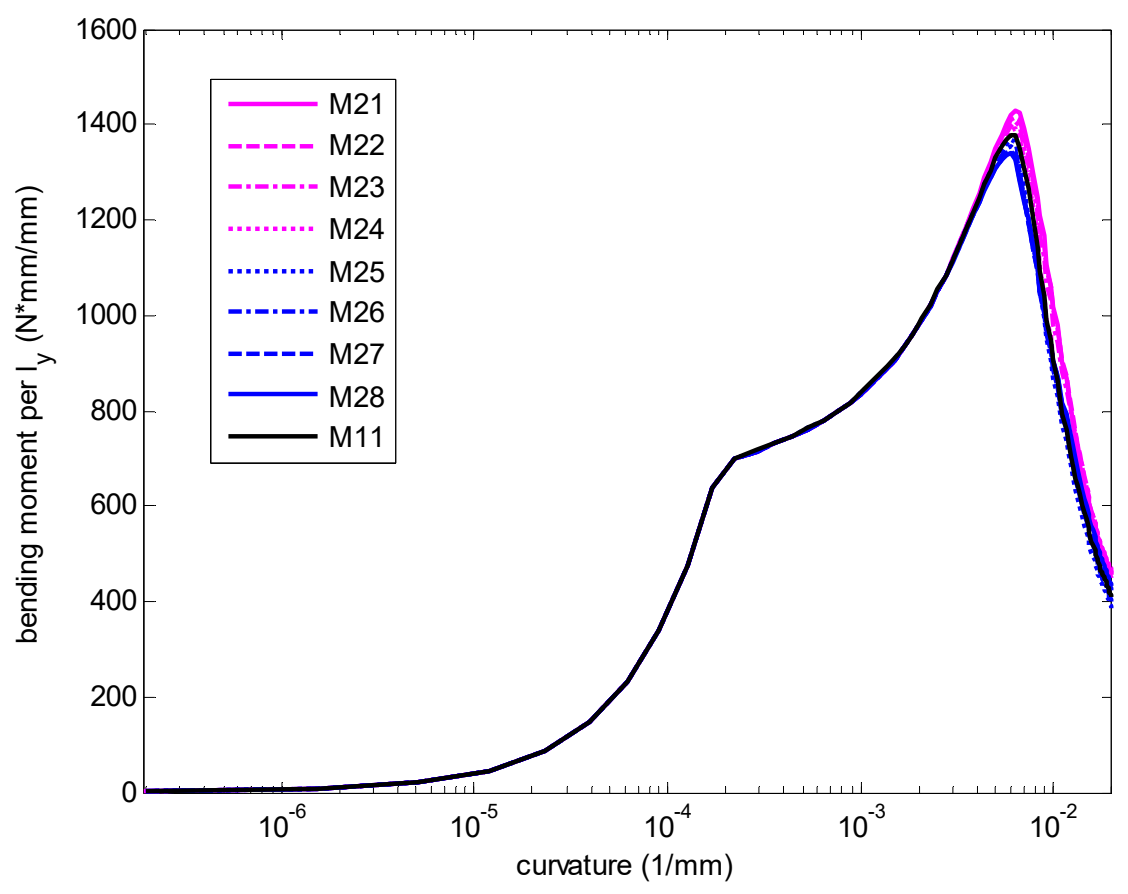

(b) 
Figure 22

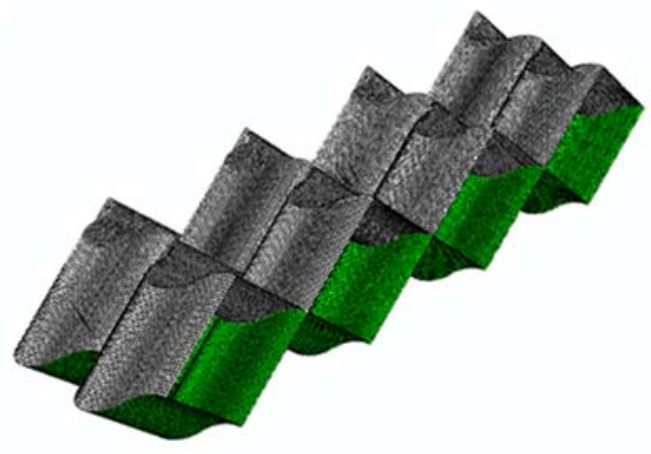

M31

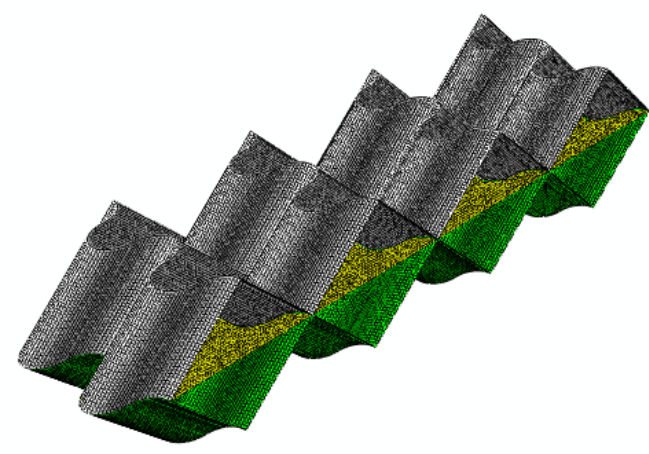

M32 
Figure 23

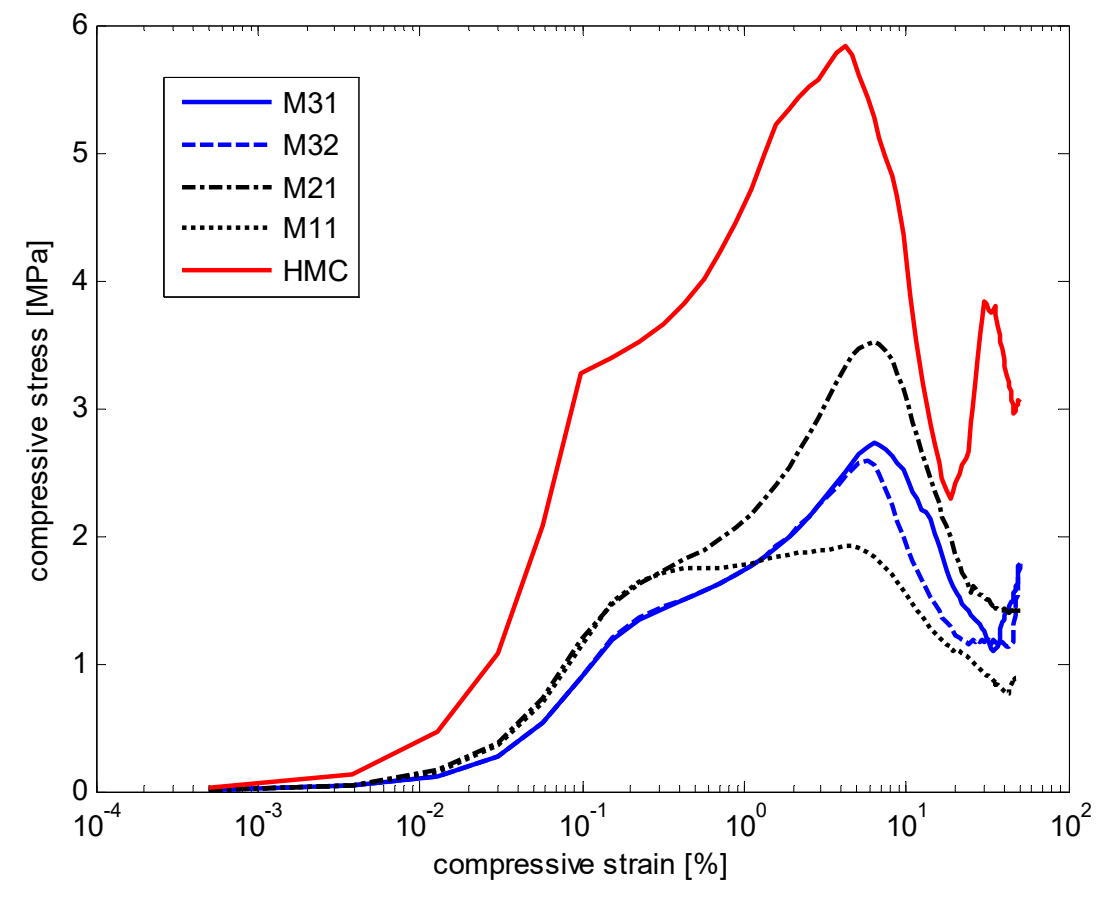


Figure 24

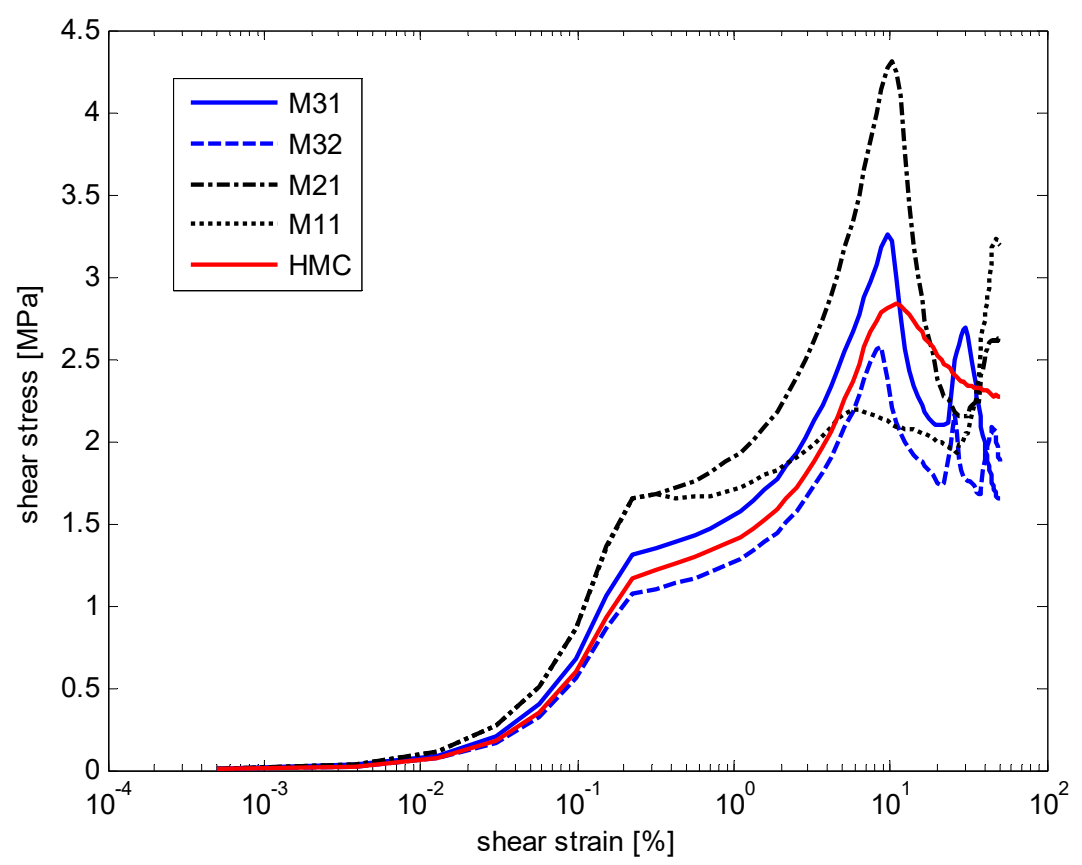

(a)

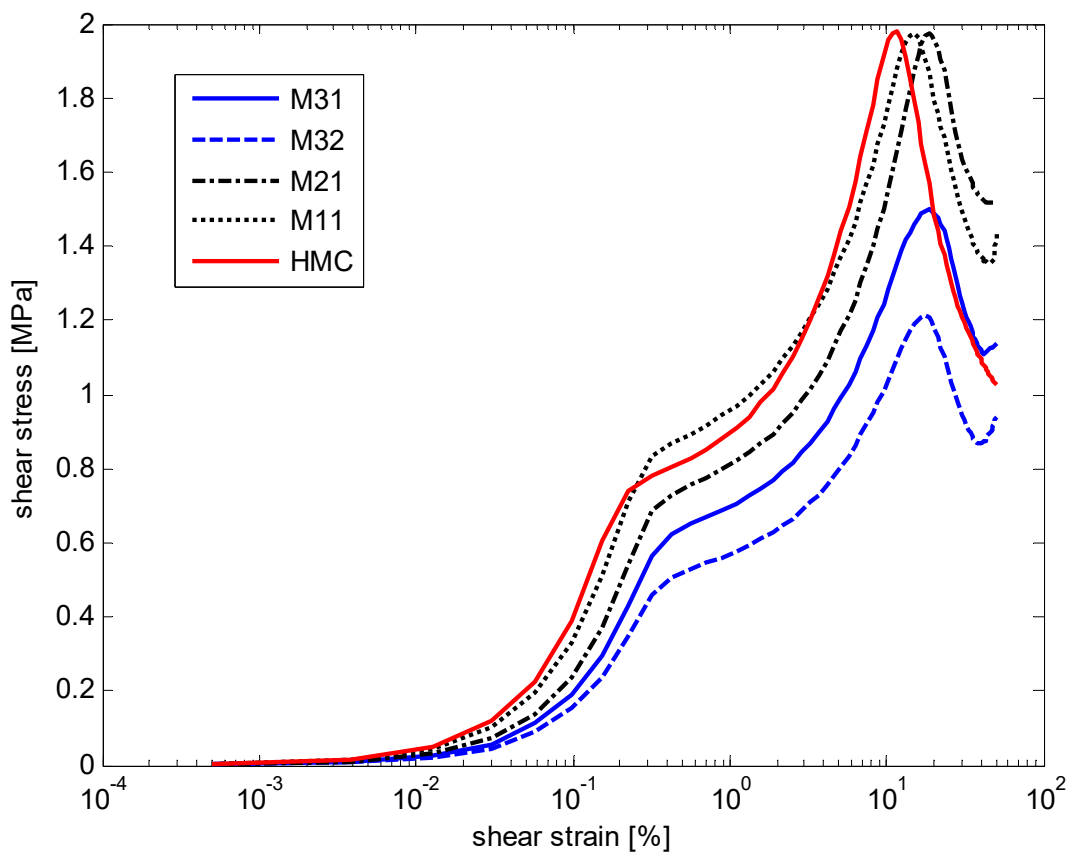

(b) 
Figure 25

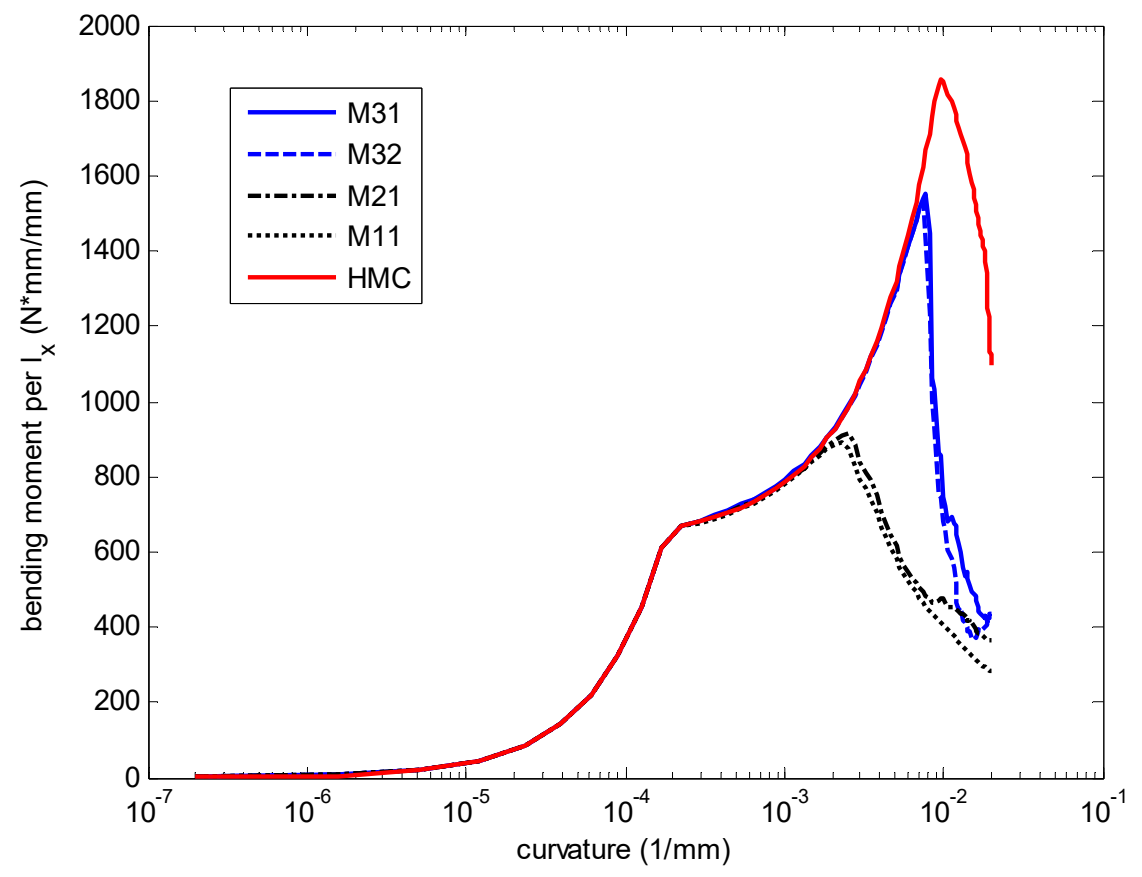

(a)

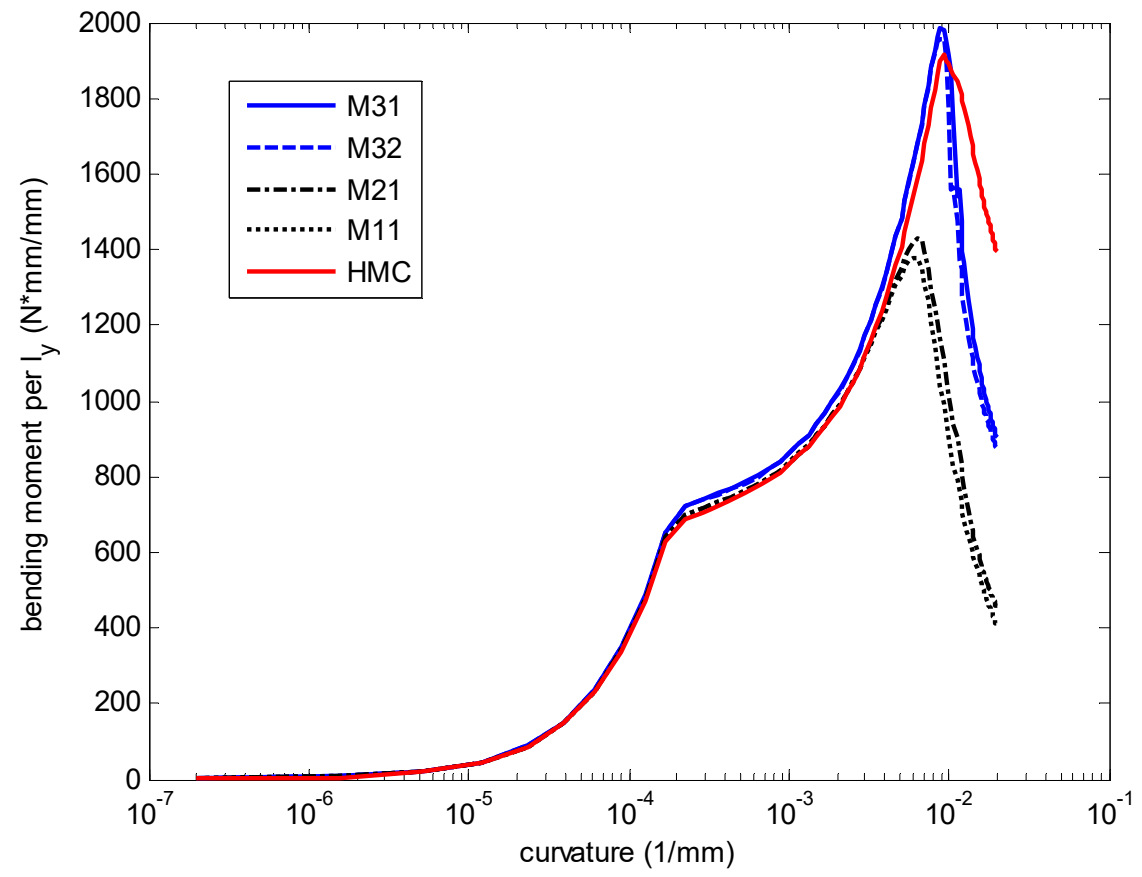

(b) 
Figure 26

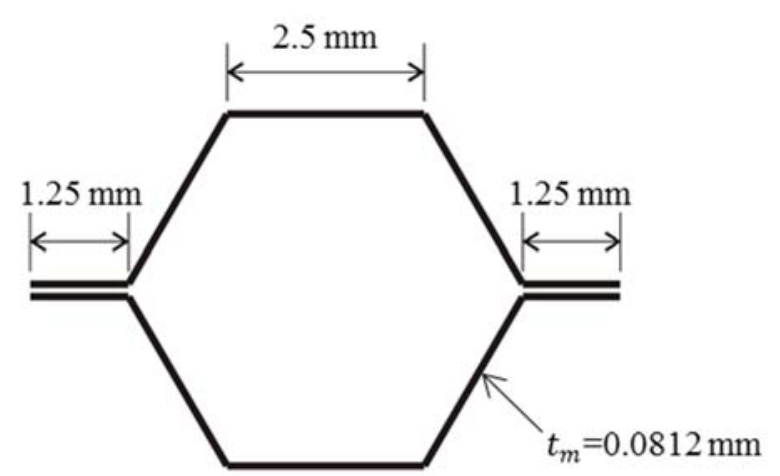

(a)

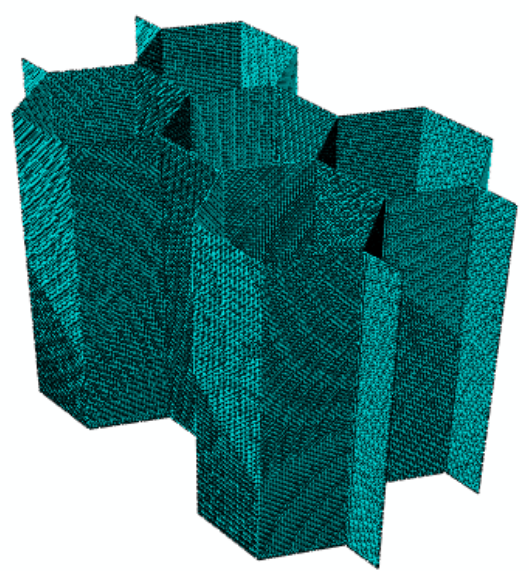

(b) 
Table 1

\begin{tabular}{|c|c|c|c|c|c|c|c|}
\hline Model & $\begin{array}{c}\alpha \\
{[\mathrm{rad}]}\end{array}$ & $\begin{array}{c}h_{x} \\
{[\mathrm{~mm}]}\end{array}$ & $\begin{array}{c}\beta \\
{[\mathrm{rad}]}\end{array}$ & $\begin{array}{c}h_{y} \\
{[\mathrm{~mm}]}\end{array}$ & $\begin{array}{c}H_{c} \\
{[\mathrm{~mm}]}\end{array}$ & $\begin{array}{c}S_{u} \\
{\left[\mathrm{~mm}^{2}\right]}\end{array}$ & $\begin{array}{c}t_{m} \\
{[\mathrm{~mm}]}\end{array}$ \\
\hline M11 & $\pi / 4$ & 5 & $\pi / 4$ & 10 & 10 & 200 & 0.25 \\
\hline M12 & $\pi / 4$ & 10 & $\pi / 4$ & 10 & 10 & 400 & 0.25 \\
\hline M13 & $\pi / 3$ & 5 & $\pi / 4$ & 10 & 10 & 115.47 & 0.1768 \\
\hline M14 & $\pi / 3$ & 10 & $\pi / 4$ & 10 & 10 & 230.94 & 0.1768 \\
\hline M15 & $\pi / 4$ & 5 & $\pi / 3$ & 10 & 10 & 115.47 & 0.1768 \\
\hline M16 & $\pi / 4$ & 10 & $\pi / 3$ & 10 & 10 & 230.94 & 0.1768 \\
\hline M17 & $\pi / 3$ & 5 & $\pi / 3$ & 10 & 10 & 66.67 & 0.125 \\
\hline M18 & $\pi / 3$ & 10 & $\pi / 3$ & 10 & 10 & 133.33 & 0.125 \\
\hline
\end{tabular}


Table 2

\begin{tabular}{|c|c|c|c|c|c|}
\hline Material & $\rho_{m}\left[\mathrm{~kg} / \mathrm{m}^{3}\right]$ & $E[\mathrm{GPa}]$ & $\sigma_{y}[\mathrm{GPa}]$ & $\sigma_{u t s}[\mathrm{GPa}]$ & $v$ \\
\hline $5052-\mathrm{O} \mathrm{Al}$ & 2690 & 69.6 & 65.5 & 193 & 0.33 \\
\hline
\end{tabular}


Table 3

\begin{tabular}{|c|c|c|c|}
\hline Model & $E_{c}[\mathrm{MPa}]$ & $E_{s 1}[\mathrm{MPa}]$ & $E_{s 2}[\mathrm{MPa}]$ \\
\hline M11 & 1202 & 874 & 337 \\
\hline M12 & 1562 & 879 & 470 \\
\hline M13 & 1252 & 884 & 166 \\
\hline M14 & 1806 & 911 & 253 \\
\hline M15 & 2268 & 735 & 466 \\
\hline M16 & 2519 & 784 & 554 \\
\hline M17 & 2308 & 762 & 229 \\
\hline M18 & 2667 & 864 & 285 \\
\hline
\end{tabular}


Table 4

\begin{tabular}{|c|c|c|c|c|c|c|}
\hline Model & $\begin{array}{c}E I_{x} \\
{\left[\mathrm{kN} \cdot \mathrm{mm}^{2} / \mathrm{mm}\right]}\end{array}$ & $\begin{array}{c}M_{x}^{c r} \\
{[\mathrm{~N} \cdot \mathrm{mm} / \mathrm{mm}]}\end{array}$ & $\begin{array}{c}k_{x}^{c r} \\
{\left[\mathrm{~mm}^{-1}\right]}\end{array}$ & $\begin{array}{c}E I_{y} \\
{\left[\mathrm{kN} \cdot \mathrm{mm}^{2} / \mathrm{mm}\right]}\end{array}$ & $\begin{array}{c}M_{y}^{c r} \\
{[\mathrm{~N} \cdot \mathrm{mm} / \mathrm{mm}]}\end{array}$ & $\begin{array}{c}k_{y}^{c r} \\
{\left[\mathrm{~mm}^{-1}\right]}\end{array}$ \\
\hline M11 & 3575 & 894 & 0.0023 & 3757 & 1378 & 0.0063 \\
\hline M12 & 3628 & 1263 & 0.0053 & 3664 & 1236 & 0.0053 \\
\hline M13 & 3583 & 914 & 0.0025 & 3816 & 1691 & 0.0074 \\
\hline M14 & 3636 & 1518 & 0.0071 & 3740 & 1399 & 0.0063 \\
\hline M15 & 3588 & 1599 & 0.0078 & 3676 & 1767 & 0.0096 \\
\hline M16 & 3655 & 1508 & 0.0067 & 3608 & 1358 & 0.0063 \\
\hline M17 & 3583 & 1690 & 0.0085 & 3750 & 1907 & 0.0096 \\
\hline M18 & 3636 & 1544 & 0.0074 & 3664 & 1475 & 0.0067 \\
\hline
\end{tabular}


Table 5

\begin{tabular}{|c|c|c|c|c|}
\hline Model & $\gamma[\mathrm{rad}]$ & $H_{c}[\mathrm{~mm}]$ & $S_{u}\left[\mathrm{~mm}^{2}\right]$ & $t_{m}[\mathrm{~mm}]$ \\
\hline M21 & $\pi / 4$ & 10 & 200 & 0.2251 \\
\hline M22 & $\arctan (3 / 4)$ & 10 & 200 & 0.2331 \\
\hline M23 & $\arctan (1 / 2)$ & 10 & 200 & 0.2411 \\
\hline M24 & $\arctan (1 / 4)$ & 10 & 200 & 0.2475 \\
\hline M25 & $-\arctan (1 / 4)$ & 10 & 200 & 0.2475 \\
\hline M26 & $-\arctan (1 / 2)$ & 10 & 200 & 0.2411 \\
\hline M27 & $-\arctan (3 / 4)$ & 10 & 200 & 0.2331 \\
\hline M28 & $-\pi / 4$ & 10 & 200 & 0.2251 \\
\hline
\end{tabular}


Table 6

\begin{tabular}{|c|c|c|c|c|c|c|}
\hline Model & $E_{c}[\mathrm{MPa}]$ & $\sigma_{c}^{c r}[\mathrm{MPa}]$ & $E_{s 1}[\mathrm{MPa}]$ & $\sigma_{s 1}^{c r}[\mathrm{MPa}]$ & $E_{s 2}[\mathrm{MPa}]$ & $\sigma_{s 2}^{c r}[\mathrm{MPa}]$ \\
\hline M21 & 1214 & 3.517 & 881 & 4.314 & 241 & 1.973 \\
\hline M22 & 1201 & 3.288 & 880 & 3.916 & 259 & 2.034 \\
\hline M23 & 1185 & 2.831 & 878 & 2.939 & 284 & 2.001 \\
\hline M24 & 1169 & 2.220 & 876 & 2.297 & 314 & 1.842 \\
\hline M11 & 1153 & 1.916 & 874 & 2.186 & 336 & 1.974 \\
\hline M25 & 1115 & 2.070 & 873 & 2.549 & 323 & 1.961 \\
\hline M26 & 1079 & 2.405 & 873 & 2.944 & 301 & 2.081 \\
\hline M27 & 1053 & 2.798 & 874 & 3.359 & 282 & 2.086 \\
\hline M28 & 1034 & 3.157 & 876 & 3.917 & 267 & 2.086 \\
\hline
\end{tabular}


Table 7

\begin{tabular}{|c|c|c|c|c|}
\hline Model & $\begin{array}{c}E I_{x} \\
{\left[\mathrm{kN} \cdot \mathrm{mm}^{2} / \mathrm{mm}\right]}\end{array}$ & $\begin{array}{c}M_{x}^{c r} \\
{[\mathrm{~N} \cdot \mathrm{mm} / \mathrm{mm}]}\end{array}$ & $\begin{array}{c}E I_{y} \\
{\left[\mathrm{kN} \cdot \mathrm{mm}^{2} / \mathrm{mm}\right]}\end{array}$ & $\begin{array}{c}M_{y}^{c r} \\
{[\mathrm{~N} \cdot \mathrm{mm} / \mathrm{mm}]}\end{array}$ \\
\hline M21 & 3597 & 913 & 3762 & 1427 \\
\hline M22 & 3596 & 905 & 3761 & 1416 \\
\hline M23 & 3595 & 900 & 3759 & 1404 \\
\hline M24 & 3594 & 898 & 3760 & 1391 \\
\hline M11 & 3586 & 894 & 3763 & 1380 \\
\hline M25 & 3585 & 881 & 3763 & 1368 \\
\hline M26 & 3581 & 868 & 3762 & 1354 \\
\hline M27 & 3579 & 854 & 3762 & 1343 \\
\hline M28 & 3579 & 851 & 3763 & 1339 \\
\hline
\end{tabular}


Table 8

\begin{tabular}{|c|c|c|c|c|}
\hline Model & Number of layers & $H_{c}[\mathrm{~mm}]$ & $S_{u}\left[\mathrm{~mm}^{2}\right]$ & $t_{m}[\mathrm{~mm}]$ \\
\hline M31 & 2 & 10 & 50 & 0.11254 \\
\hline M32 & 3 & 10 & 50 & 0.09186 \\
\hline
\end{tabular}

